Research Article

\title{
Supervariable and BRST Approaches to a Reparameterization Invariant Nonrelativistic System
}

\author{
A. K. Rao ${ }^{D},{ }^{1}$ A. Tripathi, ${ }^{1}$ and R. P. Malik $\mathbb{D}^{1,2}$ \\ ${ }^{1}$ Physics Department, Institute of Science, Banaras Hindu University, Varanasi, 221005 (U.P.), India \\ ${ }^{2}$ DST Centre for Interdisciplinary Mathematical Sciences, Institute of Science, Banaras Hindu University, Varanasi 221005, India
}

Correspondence should be addressed to R. P. Malik; rpmalik1995@gmail.com

Received 8 February 2021; Revised 3 June 2021; Accepted 24 June 2021; Published 23 July 2021

Academic Editor: Mariana Frank

Copyright ( 2021 A. K. Rao et al. This is an open access article distributed under the Creative Commons Attribution License, which permits unrestricted use, distribution, and reproduction in any medium, provided the original work is properly cited. The publication of this article was funded by SCOAP ${ }^{3}$.

\begin{abstract}
We exploit the theoretical strength of the supervariable and Becchi-Rouet-Stora-Tyutin (BRST) formalisms to derive the proper (i.e., off-shell nilpotent and absolutely anticommuting) (anti-)BRST symmetry transformations for the reparameterization invariant model of a nonrelativistic (NR) free particle whose space $(x)$ and time $(t)$ variables are a function of an evolution parameter $(\tau)$. The infinitesimal reparameterization (i.e., 1D diffeomorphism) symmetry transformation of our theory is defined w.r.t. this evolution parameter $(\tau)$. We apply the modified Bonora-Tonin (BT) supervariable approach (MBTSA) as well as the (anti)chiral supervariable approach (ACSA) to BRST formalism to discuss various aspects of our present system. For this purpose, our 1D ordinary theory (parameterized by $\tau$ ) is generalized onto a $(1,2)$-dimensional supermanifold which is characterized by the superspace coordinates $Z^{M}=(\tau, \theta, \bar{\theta})$ where a pair of the Grassmannian variables satisfy the fermionic relationships: $\theta^{2}=\bar{\theta}^{2}=0, \theta \bar{\theta}+\bar{\theta} \theta=0$, and $\tau$ is the bosonic evolution parameter. In the context of ACSA, we take into account only the $(1,1)$-dimensional (anti)chiral super submanifolds of the general $(1,2)$-dimensional supermanifold. The derivation of the universal Curci-Ferrari- (CF-) type restriction, from various underlying theoretical methods, is a novel observation in our present endeavor. Furthermore, we note that the form of the gauge-fixing and Faddeev-Popov ghost terms for our NR and nonSUSY system is exactly the same as that of the reparameterization invariant SUSY (i.e., spinning) and non-SUSY (i.e., scalar) relativistic particles. This is a novel observation, too.
\end{abstract}

\section{Introduction}

During the last few years, there has been an upsurge of interest in the study of diffeomorphism invariant theories because one of the key and decisive features of the gravitational and (super)string theories is the observation that they respect the classical diffeomorphism symmetry transformations. The latter symmetry transformations can be exploited within the framework of Becchi-Rouet-Stora-Tyutin (BRST) formalism [1-4] where the classical diffeomorphism symmetry transformation is elevated to the quantum (anti-)BRST symmetry transformations. In fact, it is the key feature of the BRST formalism that the classical diffeomorphism transformation parameter is traded with the fermionic (anti)ghost fields/variables at the quantum level. In other words, the (anti-)BRST transformations are of the supersymmetric (SUSY) kind under which the bosonic type of fields/variables transforms to the fermionic type of fields/variables and vice versa. Two of the key properties of the (anti-)BRST transformations are the on-shell/off-shell nilpotency and absolute anticommutativity. These key properties encompass in their folds the fermionic as well as independent natures of the quantum BRST and anti-BRST symmetries at the level of physical interpretation. The nilpotency property (i.e., fermionic nature) of the (anti-)BRST symmetries (and their corresponding charges) is also connected with some aspects of the cohomological properties of differential geometry and a few decisive features of supersymmetry. 
The BRST formalism has been exploited in the covariant canonical quantization of the gauge and diffeomorphism invariant theories in the past. At the classical level, the gauge theories are characterized by the existence of the first-class constraints $[5,6]$ on them. This fundamental feature is translated, at the quantum level, into the language of the existence of the Curci-Ferrari- (CF-) type restriction(s) when the classical theory is quantized by exploiting the theoretical richness of BRST formalism. Hence, the existence of the CF-type restriction(s) is the key signature of a BRST-quantized version of the gauge and/or diffeomorphism invariant theory. The CF-type restrictions are (i) deeply connected with the geometrical objects called gerbes $[7,8]$, (ii) responsible for the absolute anticommutativity of the quantum (anti-)BRST transformations, and (iii) the root cause behind the existence of the coupled (but equivalent) Lagrangians/Lagrangian densities for the (anti-)BRST invariant quantum theories (corresponding to the classical gauge/diffeomorphism invariant theories). The Abelian 1-form gauge theory is an exception where we obtain a unique (anti-)BRST invariant Lagrangian density because the CF-type restriction is trivial in this case. However, this restriction turns out to be the limiting case of the non-Abelian 1-form gauge theory where the nontrivial CF condition exists [9].

It is the supervariable/superfield approaches [10-21] to BRST formalism that provide the geometrical basis for the off-shell nilpotency and absolute anticommutativity of the (anti-)BRST symmetries as well as the existence of the CFtype restrictions for a BRST-quantized gauge/diffeomorphism invariant theory. In the usual superfield approaches (USFA), it is the horizontality condition (HC) that plays a decisive role as it leads to (i) the derivation of the (anti-)BRST symmetry transformations for only the gauge and (anti)ghost fields as well as (ii) the derivation of the CF-type restriction(s). The augmented version of the superfield approach (AVSA) is an extension of USFA where, in addition to the $\mathrm{HC}$, the gauge (i.e., (anti-)BRST) invariant restrictions are exploited together which lead to the derivation of the (anti)BRST symmetry transformations for the gauge, (anti)ghost, and matter fields together in an interacting gauge theory. It has been a challenging problem to incorporate the diffeomorphism transformation within the ambit of the superfield approach to gauge theories (see, e.g., [14-16]) so that one can discuss the gravitational and (super)string theories within the framework of USFA/AVSA. In this direction, a breakthrough has recently been made by Bonora [22] where the superfield approach has been applied to derive the proper (anti-)BRST transformations as well as the CF-type restriction for the $D$-dimensional diffeomorphism invariant theory. This approach has been christened by us as the modified Bonora-Tonin (BT) superfield approach (MBTSA) to BRST formalism. In a recent couple of papers [23, 24], we have applied the theoretical beauty of the MBTSA as well as ACSA (i.e., (anti)chiral superfield/supervariable approach) to BRST formalism [25-29] in the context of the 1D diffeomorphism (i.e., reparameterization) invariant theories of the non-SUSY (i.e., scalar) as well as SUSY (i.e., spinning) relativistic free particles.
The central theme of our present investigation is to concentrate on the reparameterization (i.e., 1D diffeomorphism) invariant theory of a massive nonsupersymmetric (NSUSY) and nonrelativistic (NR) free particle where the standard NR Lagrangian $L_{0}^{(t)}(x, \dot{x})=(1 / 2) m \dot{x}^{2}$ (with $\dot{x}=d x / d t$ ) is rendered reparameterization invariant by treating the "time" variable on a par with the $x$ variable [30] parameterized by an evolution parameter $\tau$ such that the new Lagrangian $L_{0}^{(\tau)}(x, \dot{x}, t, \dot{t})=m \dot{x}^{2} / 2 \dot{t}$, where $\dot{x}=d x /$ $d \tau$ and $\dot{t}=d t / d \tau$. The latter Lagrangian respects the reparameterization symmetry $[31,32]$, and it has been discussed in different theoretical settings where the noncommutativity of the spacetime appears by the symmetry considerations, constraint analysis, redefinitions of variables, etc. This reparameterization invariant model of the free particle $\left(\dot{p}_{x}=\dot{p}_{t}=0\right)$ has been discussed by us within the frameworks of BRST formalism as well as quantum groups [32]. However, in the BRST analysis, we have exploited the gauge symmetry of this NSUSY and NR system [32] without discussing anything about the reparameterization transformations. In our present investigation, we have applied the beautiful blend of theoretical ideas from MBTSA and ACSA to derive the proper (anti-)BRST symmetries and CF-type restriction for this NR system. This model is interesting in its own right as it is a NR system (unlike our earlier discussions [23, 24] on the relativistic systems), and "time" itself has been treated as a physical observable that depends on the evolution parameter $\tau$. The latter property of our present NR system is important as "time" has also been treated as an observable in quantum mechanics instead of an evolution parameter (see, e.g., [30]).

The following motivating factors have been at the heart of our curiosity in pursuing our present endeavor. First, so far, we have been able to apply the beautiful blend of theoretical ideas behind MBTSA and ACSA to BRST formalism in the cases of reparameterization invariant systems of the (i) relativistic nonsupersymmetric (NSUSY) scalar free particle and (ii) supersymmetric (SUSY) (i.e., spinning) relativistic free particle. Thus, it has been a challenging problem for us to apply the same theoretical ideas to discuss the NSUSY and nonrelativistic (NR) system of a reparameterization invariant free particle. We have accomplished this goal in our present investigation. Second, we have shown the universality of the CF-type restriction in the cases of reparameterization (i.e., 1D diffeomorphism) invariant NSUSY as well as SUSY systems of the free relativistic particles. Thus, we have been motivated to see the existence of the same CF-type restriction in our present case of reparameterization invariant systems of NSUSY and NR free particles. We have been able to demonstrate that it is the same CF-type restriction that exists in the BRST approach to our present NSUSY and NR system. Third, we have found out that the gaugefixing and Faddeev-Popov (FP) ghost terms for the systems of non-SUSY (i.e., scalar) and SUSY (i.e., spinning) relativistic particles are the same within the ambit of BRST formalism. Thus, we have been curious to find out the gaugefixing and FP ghost terms for our present non-SUSY and 
NR system. It is surprising that the above terms are the same for our present system, too. Finally, our present work is another modest initial step towards our main goal of applying the theoretical potential of MBTSA and ACSA to the physical four $(3+1)$-dimensional (4D) diffeomorphism invariant gravitational and (super)string theories in the higher dimensions (i.e., $D>4$ ) of spacetime.

The contents of our present endeavor are organized as follows. In Section 2, we recapitulate the bare essentials of the Lagrangian formulation of our reparameterization invariant nonrelativistic system and discuss the BRST quantization of this model by exploiting its infinitesimal gauge symmetry transformations. Section 3 is devoted to the application of MBTSA for the derivation of (i) the quantum (anti-)BRST symmetry transformations for the phase space variables and (ii) the underlying (anti-)BRST invariant Curci-Ferrari(CF-) type restriction (corresponding to the classical infinitesimal reparameterization symmetry transformations). The theoretical content of Section 4 is related to the derivation of the full set of (anti-)BRST symmetry transformations by requiring the off-shell nilpotency and absolute anticommutativity properties. We also show the existence of the CF-type restriction and deduce the coupled (anti-)BRST invariant Lagrangians for our theory. In Section 5, we derive the (anti-)BRST symmetry transformations for the other variables (i.e., different from the phase variables) within the purview of ACSA. Section 6 deals with the proof of the equivalence of the coupled Lagrangians within the framework of ACSA to BRST formalism. In Section 7, we prove the offshell nilpotency and absolute anticommutativity of the conserved (anti-)BRST charges in the ordinary space and superspace (by exploiting the theoretical richness of ACSA to BRST formalism). Finally, in Section 8, we discuss our key results and point out a few future directions for further investigation(s).

In Appendices A, B, and $\mathrm{C}$, we perform some explicit computations that supplement as well as corroborate the key claims that have been made in the main body of our text.

1.1. Convention and Notations. We adopt the convention of the left derivative w.r.t. all the fermionic variables of our theory. In the whole body of the text, we denote the fermionic (anti-)BRST transformations by the symbols $s_{(a) b}$ and corresponding conserved and nilpotent charges carry the notations $Q_{(a) b}$ and $Q_{(\bar{B}) B}$ in different contexts. The general $(1,2)$-dimensional supermanifold is parameterized by $Z^{M}=(\tau, \theta, \bar{\theta})$, and its chiral and antichiral super submanifolds are characterized by $(\tau, \theta)$ and $(\tau, \bar{\theta})$, respectively, where the bosonic coordinate is represented by the evolution parameter $(\tau)$ and the Grassmannian variables $(\theta, \bar{\theta})$ obey the fermionic relationships: $\theta^{2}=0, \bar{\theta}^{2}=0$, and $\theta \bar{\theta}+\bar{\theta} \theta=0$.

\section{Preliminaries: Lagrangian Formulation}

Our present section is divided into two parts. In Section 2.1, we discuss the classical infinitesimal reparameterization and gauge symmetry transformations. Section 2.2 deals with the
BRST quantization of our system by exploiting the classical gauge symmetry transformations (which are also infinitesimal and continuous).

2.1. Classical Infinitesimal Symmetries. We begin with the three equivalent reparameterization invariant Lagrangians for the free nonrelativistic and non-SUSY particle as (see, e.g., [32] for details)

$$
\begin{aligned}
L_{0}(x, \dot{x}, t, \dot{t}) & =\frac{m \dot{x}^{2}}{2 \dot{t}}, \\
L_{\mathrm{f}}\left(x, \dot{x}, t, \dot{t}, p_{x}, p_{t}\right) & =p_{x} \dot{x}+p_{t} \dot{t}-\frac{1}{2} E\left(p_{x}^{2}+2 m p_{t}\right), \\
L_{\mathrm{s}}(x, \dot{x}, t, \dot{t}) & =\frac{\dot{x}^{2}}{2 E}+\frac{m \dot{x}^{2}}{2 \dot{t}}\left[\frac{E m}{\dot{t}}-1\right],
\end{aligned}
$$

where the trajectory of the free nonrelativistic particle is embedded in a $2 \mathrm{D}$ configuration space characterized by the coordinates $[x(\tau), t(\tau)]$ and the parameter $\tau$ specifies the trajectory of the particle as an evolution parameter. The momenta variables $\left(p_{x}, p_{t}\right)$ are defined by $p_{x}=(\partial L / \partial \dot{x})$ and $p_{t}=(\partial L / \partial \dot{t})$, where $\dot{x}=(d x / d \tau)$ and $\dot{t}=(d t / d \tau)$ are the generalized "velocities" w.r.t. the coordinates $[x(\tau), t(\tau)]$ and $L$ stands for any of the three Lagrangians of Equation (1). It is self-evident that the $4 \mathrm{D}$ phase space, corresponding to the $2 \mathrm{D}$ configuration space, is characterized by $[x(\tau)$, $\left.t(\tau), p_{x}(\tau), p_{t}(\tau)\right]$. In the above Equation (1), the mass of the nonrelativistic particle is denoted by $m$ and $E(\tau)$ is the Lagrange multiplier variable that incorporates the constraint $p_{x}^{2}+2 m p_{t} \approx 0$ in the Lagrangians $L_{\mathrm{f}}$ and $L_{\mathrm{s}}$. It is straightforward to note that $L_{0}$ and $L_{\mathrm{s}}$ contain variables (and their first-order derivative) in the denominator but $L_{\mathrm{f}}$ (i.e., the first-order Lagrangian) does not incorporate any variable (and/or its derivative) in its denominator. Furthermore, the starting Lagrangian (For a free massive NR particle, the standard action integral is $S_{t}=\int_{-\infty}^{+\infty} d t\left((1 / 2) m \dot{x}^{2}\right)$, where $\dot{x}=(d x / d t)$ and time " $t$ " is the evolution parameter. This action has no reparameterization invariance. If the evolution parameter is $\tau$, then the action integral is $S_{\tau}=(m / 2) \int_{-\infty}^{+\infty} d \tau(d t / d \tau)(d x / d \tau)(d \tau / d t)$ $(d x / d \tau)(d \tau / d t)$ which leads $[31,32]$ to the final action integral as $S_{\tau}=(m / 2) \int_{-\infty}^{+\infty} d \tau\left[(d x / d \tau)^{2}(d \tau / d t)\right] \equiv \int_{-\infty}^{+\infty} d \tau L_{0}$. Hence, the starting Lagrangian becomes $L_{0}(x, \dot{x}, t, \dot{t})=m \dot{x}^{2} / 2 \dot{t}$, where $\dot{x}=(d x / d \tau)$ and $\dot{t}=(d t / d \tau)$.) $L_{0}$ does not permit the massless $(m=0)$ limit but the massless $(m=0)$ limits are well defined for $L_{\mathrm{f}}$ and the second-order Lagrangian $L_{\mathrm{s}}$. We would like to stress that the Lagrange multiplier variable behaves like the "gauge" variable due to its transformation property.It will be worthwhile to dwell a bit on the derivation of the top entry (i.e., $\left.L_{0}(x, t, \dot{x}, t)\right)$ in Equation (1). For a free massive NR particle, the standard action integral is $S_{t}=\int_{-\infty}^{+\infty}$ $d t\left(1 / 2 m \dot{x}^{2}\right)$, where $\dot{x}=(d x / d t)$ and time " $t$ " is the evolution parameter. This action has no repa-rameterization invariance. If the evolution parameter is $\tau$, then the action integral is $S_{\tau}=(m / 2) \int_{-\infty}^{+\infty} d \tau(d t / d \tau)(d x / d \tau)(d \tau / d t)(d x / d \tau)(d \tau / d t)$ which leads $[31,32]$ to the $f$-nal action integral as $S_{\tau}=(\mathrm{m} / 2)$ 
$\int_{-\infty}^{+\infty} d \tau\left[(d x / d \tau)^{2}(d \tau / d t)\right] \equiv \int_{-\infty}^{+\infty} d \tau L_{0}$. Hence, the starting Lagrangian becomes $L_{0}(x, \dot{x}, t, \dot{t})=m \dot{x}^{2} / 2 \dot{t}$ where $\dot{x}=(d x / d \tau)$ and $\dot{t}=(d t / d \tau)$.

For our further discussions, we shall concentrate on the first-order Lagrangian $L_{\mathrm{f}}$ because it has the maximum number of variables (i.e., $x, p_{x}, t, p_{t}$, and $E$ ) and allows the massless limit and there are no variables (and/or their first-order derivative w.r.t. $\tau$ ) in its denominator. It is straightforward to check that under the following infinitesimal and continuous 1D diffeomorphism (i.e., reparameterization) symmetry transformations $\left(\delta_{\mathrm{r}}\right)$, namely,

$$
\begin{aligned}
\delta_{\mathrm{r}} x & =\varepsilon \dot{x}, \\
\delta_{\mathrm{r}} p_{x} & =\varepsilon \dot{p}_{x}, \\
\delta_{\mathrm{r}} t & =\varepsilon \dot{t}, \\
\delta_{\mathrm{r}} p_{t} & =\varepsilon \dot{p}_{t}, \\
\delta_{\mathrm{r}} E & =\frac{d}{d \tau}(\varepsilon E), \\
\delta_{\mathrm{r}} L_{\mathrm{f}} & =\frac{d}{d \tau}\left(\varepsilon L_{\mathrm{f}}\right),
\end{aligned}
$$

the action integral $S=\int_{-\infty}^{+\infty} d \tau L_{\mathrm{f}}$ remains invariant (i.e., $\delta_{\mathrm{r}} S=0$ ) for the physically well-defined variables in $L_{\mathrm{f}}$ and the infinitesimal diffeomorphism transformation parameter $\varepsilon(\tau)$ in $\tau \longrightarrow \tau^{\prime}=g(\tau)=\tau-\varepsilon(\tau)$, where $g(\tau)$ is a physically well-defined function of $\tau$ such that it is finite at $\tau=0$ and vanishes off at $\tau= \pm \infty$. In fact, the infinitesimal and continuous reparameterization symmetry transformation $\left(\delta_{\mathrm{r}}\right)$ is defined as $\delta_{\mathrm{r}} \phi(\tau)=\phi^{\prime}(\tau)-\phi(\tau)$ for the generic variable $\phi(\tau)=x(\tau), p_{x}(\tau), t(\tau), p_{t}(\tau), E(\tau)$ of our present theory.

The above infinitesimal and continuous reparameterization symmetry transformations (2) encompass in their folds the gauge symmetry transformations $\left(\delta_{\mathrm{g}}\right)$ which are generated (see, e.g., [32] for details) by the first-class constraints $\Pi_{E} \approx 0,\left(p_{x}^{2}+2 m p_{t}\right) \approx 0$, where $\Pi_{E}$ is the canonical conjugate momentum corresponding to the variable $E(\tau)$. Using the following Euler-Lagrange equations of motion (ELEOMs) from $L_{\mathrm{f}}$, namely,

$$
\begin{aligned}
& \dot{p}_{x}=0, \\
& \dot{p}_{t}=0, \\
& \dot{x}=E p_{x}, \\
& \dot{t}=E m,
\end{aligned}
$$

and identifying the transformation parameters $\varepsilon(\tau) E(\tau)=\xi$ $(\tau)$, we obtain the infinitesimal and continuous gauge symmetry transformations $\left(\delta_{\mathrm{g}}\right)$, from the infinitesimal and continuous reparameterization symmetry transformations (2), as follows:

$$
\begin{gathered}
\delta_{\mathrm{g}} x=\xi p_{x}, \\
\delta_{\mathrm{g}} t=\xi m, \\
\delta_{\mathrm{g}} p_{x}=0, \\
\delta_{\mathrm{g}} p_{t}=0, \\
\delta_{\mathrm{g}} E=\dot{\xi} .
\end{gathered}
$$

It is elementary now to check that the first-order Lagrangian $\left(L_{\mathrm{f}}\right)$ transforms to a total derivative under the infinitesimal and continuous gauge transformations $\left(\delta_{\mathrm{g}}\right)$, namely,

$$
\delta_{\mathrm{g}} L_{\mathrm{f}}=\frac{d}{d \tau}\left[\frac{\xi}{2} p_{x}^{2}\right]
$$

thereby rendering the action integral $S=\int_{-\infty}^{+\infty} d \tau L_{\mathrm{f}}$ invariant (i.e., $\delta_{\mathrm{g}} S=0$ ) under the infinitesimal and continuous gauge symmetry transformations $\left(\delta_{\mathrm{g}}\right)$.

We end this section with the following decisive comments. First, the 1D diffeomorphism (i.e., reparameterization) transformations (2) are more general than the infinitesimal and continuous gauge symmetry transformations (4). Second, the Lagrange multiplier variable $E(\tau)$ behaves like a "gauge" variable due to its transformation $\delta_{\mathrm{g}} E=\dot{\xi}$ in (4). Third, all the three Lagrangians in (1) are equivalent and all of them respect the infinitesimal and continuous gauge and reparameterization symmetry transformations [32]. Fourth, all the Lagrangians describe the free motion $\left(\dot{p}_{x}=0, \dot{p}_{t}=0\right)$ of the NR particle. Hence, our system is a nonrelativistic free $\left(\dot{p}_{x}=\dot{p}_{t}=0\right)$ particle. Fifth, the first-order Lagrangian $L_{\mathrm{f}}$ is theoretically more interesting to handle because, as pointed out earlier, it incorporates the maximum number of variables. Finally, we can exploit the reparameterization and gauge symmetry transformations (2) and (4) for the BRST quantization. Following the usual BRST prescription, we note that the (anti-)BRST symmetry transformations, corresponding to the classical reparameterization symmetry transformations (2), are

$$
\begin{aligned}
s_{a b} x & =\bar{C} \dot{x}, \\
s_{a b} p_{x} & =\bar{C} \dot{p}_{x}, \\
s_{a b} t & =\bar{C} \dot{t}, \\
s_{a b} p_{t} & =\bar{C} \dot{p}_{t}, \\
s_{a b} E & =\frac{d}{d \tau}(\bar{C} E), \\
s_{b} x & =C \dot{x}, \\
s_{b} p_{x} & =C \dot{p}_{x}, \\
s_{b} t & =C \dot{t}, \\
s_{b} p_{t} & =C \dot{p}_{t}, \\
s_{b} E & =\frac{d}{d \tau}(C E),
\end{aligned}
$$


where $(\bar{C}) C$ are the fermionic $\left(C^{2}=\bar{C}^{2}=0, C \bar{C}+\bar{C} C=0\right)$ (anti)ghost variables corresponding to the classical infinitesimal diffeomorphism transformation parameter $\varepsilon(\tau)$ (cf. Equation (2)). In an exactly similar fashion, the (anti-)BRST symmetry transformations, corresponding to the classical gauge symmetry transformations (4), are

$$
\begin{aligned}
s_{a b} x & =\bar{c} p_{x}, \\
s_{a b} p_{x} & =0, \\
s_{a b} t & =\bar{c} m, \\
s_{a b} p_{t} & =0, \\
s_{a b} E & =\dot{\bar{c}}, \\
s_{b} x & =c p_{x}, \\
s_{a b} p_{x} & =0, \\
s_{b} t & =c m, \\
s_{b} p_{t} & =0, \\
s_{b} E & =\dot{c},
\end{aligned}
$$

where the fermionic $\left(c^{2}=\bar{c}^{2}=0, c \bar{c}+\bar{c} c=0\right)$ variables $(\bar{c}) c$ are the (anti)ghost variables corresponding to the classical gauge symmetry transformation parameter $\xi(\tau)$ of Equation (4). In addition to the (anti-)BRST symmetry transformations in (6) and (7), we have the following standard (anti-)BRST transformations:

$$
\begin{gathered}
s_{a b} C=i \bar{B}, \\
s_{a b} \bar{B}=0, \\
s_{b} \bar{C}=i B, \\
s_{b} B=0, \\
s_{a b} c=i \bar{b}, \\
s_{a b} \bar{b}=0, \\
s_{b} \bar{c}=i b, \\
s_{b} b=0,
\end{gathered}
$$

where the pairs $(B, \bar{B})$ and $(b, \bar{b})$ are the NakanishiLautrup auxiliary variables in the context of the BRST quantization of our reparameterization and gauge invariant system by exploiting the classical reparameterization and gauge transformations, respectively.

\subsection{Quantum Nilpotent (Anti-)BRST Symmetries} Corresponding to the Classical Gauge Symmetry Transformations. We have listed the quantum (anti-)BRST symmetries corresponding to the classical gauge symmetry transformations (4) in our Equations (7) and (8). It is elementary to check that these quantum symmetries are offshell nilpotent $\left(s_{(a) b}^{2}=0\right)$ of order two. The requirement of the absolute anticommutativity $\left(s_{b} s_{a b}+s_{a b} s_{b}=0\right)$ leads to the restriction $b+\bar{b}=0 \Rightarrow \bar{b}=-b$. As a consequence, we have the full set of (anti-)BRST symmetry transformations (corresponding to the classical gauge symmetry transformations (4)) as follows:

$$
\begin{aligned}
s_{a b} x & =\bar{c} p_{x}, \\
s_{a b} p_{x} & =0, \\
s_{a b} t & =\bar{c} m, \\
s_{a b} p_{t} & =0, \\
s_{a b} E & =\dot{\bar{c}}, \\
s_{a b} \bar{c} & =0, \\
s_{a b} c & =-i b, \\
s_{a b} b & =0, \\
s_{b} x & =c p_{x}, \\
s_{b} p_{x} & =0, \\
s_{b} t & =c m, \\
s_{b} p_{t} & =0, \\
s_{b} E & =\dot{c}, \\
s_{b} c & =0, \\
s_{b} \bar{c} & =i b, \\
s_{b} b & =0 .
\end{aligned}
$$

It is straightforward to check that the above (anti-)BRST symmetry transformations are off-shell nilpotent $\left(s_{(a) b}^{2}=0\right)$ and absolutely anticommuting $\left(s_{b} s_{a b}+s_{a b} s_{b}=0\right)$ in nature. The (anti-)BRST invariant Lagrangian $L_{b}$ (which is the generalization of the classical $L_{\mathrm{f}}$ to its quantum level) can be written as (The structure of gauge-fixing and FP ghost terms is exactly like the Abelian 1-form $\left(A^{(1)}=d x^{\mu} A_{\mu}\right)$ gauge theory where we have the BRST invariant Lagrangian density: $L_{b}=-(1 / 4) F_{\mu \nu} F^{\mu \nu}+s_{b}\left[-i \bar{c}\left(\partial_{\mu} A^{\mu}+(b / 2)\right)\right] \equiv-(1 / 4) F_{\mu \nu} F^{\mu \nu}+$ $s_{a b}\left[-i c\left(\partial_{\mu} A^{\mu}+(b / 2)\right)\right] \equiv-(1 / 4) F_{\mu \nu} F^{\mu \nu}+s_{b} s_{a b}\left[(i / 2) A^{\mu} A_{\mu}-(1 /\right.$ 2) $\bar{c} c]$. Here, $A_{\mu}$ is the vector potential, $F_{\mu \nu}=\partial_{\mu} A_{\nu}-\partial_{\nu} A_{\mu}$ is the field strength tensor, and the rest of the symbols are the same as in Equations (10) and (11). Note that the 2-form $F^{(2)}=d A^{(1)}=(1 / 2)\left(d x^{\mu} \wedge d x^{\nu}\right) F_{\mu \nu}$ defines the field strength tensor $F_{\mu \nu}$ (where $d=d x^{\mu} \partial_{\mu}$ in $F^{(2)}=d A^{(1)}$ stands for the exterior derivative of the differential geometry).):

$$
\begin{aligned}
L_{b} & =L_{\mathrm{f}}+s_{b}\left[-i \bar{c}\left(\dot{E}+\frac{b}{2}\right)\right] \equiv L_{\mathrm{f}}+s_{a b}\left[i c\left(\dot{E}+\frac{b}{2}\right)\right] \\
& \equiv L_{\mathrm{f}}+s_{b} s_{a b}\left[\frac{i E^{2}}{2}-\frac{\bar{c} c}{2}\right] \equiv L_{\mathrm{f}}-s_{a b} s_{b}\left[\frac{i E^{2}}{2}-\frac{\bar{c} c}{2}\right] .
\end{aligned}
$$

In other words, we have expressed the gauge-fixing and Faddeev-Popov (FP) ghost terms in three different ways which, ultimately, lead to the following expression for $L_{b}$, namely, 


$$
\begin{aligned}
L_{b}= & L_{\mathrm{f}}+b \dot{E}+\frac{b^{2}}{2}-\dot{\bar{i}} \dot{c} \equiv p_{x} \dot{x}+p_{t} \dot{t}-\frac{1}{2} E\left(p_{x}^{2}+2 m p_{t}\right) \\
& +b \dot{E}+\frac{b^{2}}{2}-\dot{i \bar{c} \dot{c}} .
\end{aligned}
$$

It should be noted that we have dropped the total derivative terms in obtaining $L_{b}$ from (10). The above equation demonstrates that we have obtained a unique (anti-)BRST invariant Lagrangian. This has happened because the CF-type restriction is trivial (i.e., $b+\bar{b}=0$ ) in our simple case of the NR system. We can explicitly check that

$$
\begin{gathered}
s_{b} L_{b}=\frac{d}{d \tau}\left[\frac{c}{2} p_{x}^{2}+b \dot{\bar{c}}\right], \\
s_{a b} L_{b}=\frac{d}{d \tau}\left[\frac{\bar{c}}{2} p_{x}^{2}+b \dot{\bar{c}}\right],
\end{gathered}
$$

which lead to the derivation of the conserved (anti-)BRST charges $\left[Q_{(a) b}\right]$ as follows:

$$
\begin{aligned}
& Q_{a b}=b \dot{\bar{c}}+\frac{1}{2} \bar{c}\left(p_{x}^{2}+2 m p_{t}\right) \equiv b \dot{\bar{c}}-\dot{b} \bar{c}, \\
& Q_{b}=b \dot{c}+\frac{1}{2} c\left(p_{x}^{2}+2 m p_{t}\right) \equiv b \dot{c}-\dot{b} c .
\end{aligned}
$$

In the last step, we have used $\dot{b}=-(1 / 2)\left(p_{x}^{2}+2 m p_{t}\right)$ which emerges out as the EL-EOM from $L_{b}$ w.r.t. the Lagrange multiplier variable $E(\tau)$. The structure of gauge-fixing and FP ghost terms in Equation (10) is exactly like the Abelian 1-form $\left(A^{(1)}=d x^{\mu} A_{\mu}\right)$ gauge theory where we have the BRST invariant Lagrangian density: $\mathscr{L}_{b}=-1 / 4 F_{\mu \nu} F^{\mu v}+s_{b}\left[-i \bar{c}\left(\partial_{\mu} A^{\mu}+b /\right.\right.$ $2)] \equiv-1 / 4 F_{\mu v} F^{\mu v}+s_{a b}\left[-i c\left(\partial_{\mu} A^{\mu}+b / 2\right)\right] \equiv-1 / 4 F_{\mu v} F^{\mu v}+s_{b} s_{a b}[1$ $\left.12 A^{\mu} A_{\mu}-1 / 2 \bar{c} c\right]$. Here, $A_{\mu}$ is the vector potential, $F_{\mu \nu}=\partial_{\mu} A_{v}$ $-\partial_{\nu} A_{\mu}$ is the field strength tensor, and the rest of the symbols are the same as in Equation (10) and (11). Note that the 2form $F^{(2)}=d A^{(1)}=1 / 2\left(d x^{\mu} \Lambda d x^{\nu}\right) F_{\mu \nu} \quad$ defines the field strength tensor $F_{\mu \nu}$ (where $d=d x^{\mu} \partial_{\mu}$ in $F^{(2)}=d A^{(1)}$ which stands for the exterior derivative of the differential geometry).

We close this section with a few crucial and decisive remarks. First, we can check that the (anti-)BRST charges are conserved $\left[\dot{Q}_{(a) b}=0\right]$ by using the EL-EOMs. Second, the (anti-)BRST charges $\left[Q_{(a) b}\right]$ are off-shell nilpotent $\left[Q_{(a) b}^{2}\right.$ $=0]$ of order two due to the direct observations that $s_{b} Q_{b}$ $=s_{b}[b \dot{c}-\dot{b} c]=0 \quad$ and $s_{a b} Q_{a b}=s_{a b}[b \dot{\bar{c}}-\dot{b} \bar{c}]=0 \quad$ which encode in their folds $s_{b} Q_{b}=-i\left\{Q_{b}, Q_{b}\right\}=0 \Rightarrow Q_{b}^{2}=0$ and $s_{a b} Q_{a b}=-i\left\{Q_{a b}, Q_{a b}\right\}=0 \Rightarrow Q_{a b}^{2}=0$. Third, the above nilpotency is also encoded in $Q_{b}=s_{b}[b E+\dot{\overline{i c} c}]$ implying that $s_{b} Q_{b}$ $=0$ due to $s_{b}^{2}=0$ and we also point out that $s_{a b} Q_{a b}=0$ due to the nilpotency $\left(s_{a b}^{2}=0\right)$ of $s_{a b}$ because $Q_{a b}=s_{a b}[b E+i \bar{c} \dot{c}]$. Fourth, we observe that $s_{a b} Q_{b}=i\left\{Q_{b}, Q_{a b}\right\} \equiv-i b \dot{b}+i \dot{b} b=0$ and $s_{b} Q_{a b}=-i\left\{Q_{a b}, Q_{b}\right\}=i b \dot{b}-i b \dot{b}=0$ which explicitly lead to the conclusion that the off-shell nilpotent charges $Q_{(a) b}$ are also absolutely anticommuting $\left(Q_{b} Q_{a b}+Q_{a b} Q_{b}=0\right)$ in nature. Fifth, the above observation of the absolute anticommutativity can be also expressed in terms of the nilpotency property because we observe that $Q_{b}=s_{a b}(-i \ddot{c} c)$ and $Q_{a b}=$ $s_{b}(\dot{\overline{i c}})$ which imply that $s_{a b} Q_{b}=-i\left\{Q_{b}, Q_{a b}\right\}=0$ and $s_{b} Q_{a b}$ $=-i\left\{Q_{a b}, Q_{b}\right\}=0$ (due to the off-shell nilpotency $\left(s_{a b}^{2}=0\right.$ ) of the anti-BRST as well as the off-shell nilpotency $\left(s_{b}^{2}=0\right)$ of the BRST symmetry transformations). Sixth, it can be seen that the physical space (i.e., $\mid$ phys $>$ ) in the total Hilbert space of states is defined by $Q_{b} \mid$ phys $>=0$ which implies that $b \mid$ phys $>\equiv \Pi_{E} \mid$ phys $>=0$ and $\dot{b} \mid$ phys $>\equiv\left(p_{x}^{2}+2 m p_{t}\right) \mid$ phys $>$ $=0$. In other words, the Dirac quantization conditions (with the first-class constraints $\Pi_{E} \approx 0, p_{x}^{2}+2 m p_{t} \approx 0$ ) are beautifully satisfied. Finally, the physicality criterion $Q_{b} \mid$ phys $>=$ 0 implies that the two physical states $\mid$ phys ${ }^{\prime}>$ and $\mid$ phys $>$ belong to the same cohomological class w.r.t. the nilpotent BRST charge $Q_{b}$ if they differ by a BRST exact state (i.e., | phys $^{\prime}>=\mid$ phys $>+Q_{b} \mid \chi>$ for nonnull $\mid \chi>$ ).

\section{Nilpotent and Anticommuting (Anti-)BRST Symmetries for the Phase Variables: MBTSA}

This section is devoted to the derivation of the transformations $s_{b} x=C \dot{x}, s_{b} p_{x}=C \dot{p}_{x}, s_{b} t=C \dot{t}, s_{b} p_{t}=C \dot{p}_{t}, s_{a b} x=\bar{C} \dot{x}, s_{a b}$ $p_{x}=\bar{C} \dot{p}_{x}, s_{a b} t=\bar{C} \dot{t}$, and $s_{a b} p_{t}=\bar{C} \dot{p}_{t}$ by exploiting the theoretical tricks of MBTSA. Before we set out to perform this exercise, it is essential to pinpoint the off-shell nilpotency and absolute anticommutativity properties of the (anti-)BRST symmetry transformations on the phase variables (cf. Equation (6)). It can be easily checked that the off-shell nilpotency requirement (i.e., $s_{(a) b}^{2} S=0, S=x$, $p_{x}, t, p_{t}$ ) leads to the (anti-)BRST symmetry transformations for the (anti)ghost variables as

$$
\begin{aligned}
s_{a b} \bar{C} & =\bar{C} \dot{\bar{C}}, \\
s_{b} C & =C \dot{C} .
\end{aligned}
$$

Furthermore, the absolute anticommutativity requirement $\left\{s_{b}, s_{a b}\right\} S=0$ for the generic phase variable $S=x$, $p_{x}, t, p_{t}$ leads to the following:

$\left\{s_{b}, s_{a b}\right\} S=i[B+\bar{B}+i(\bar{C} \dot{C}-\dot{\bar{C}} C)] \dot{S} \Rightarrow B+\bar{B}+i(\bar{C} \dot{C}-\dot{\bar{C}} C)=0$.

In other words, the absolute anticommutativity property $\left(s_{b} s_{a b}+s_{a b} s_{b}=0\right)$ is satisfied if and only if we invoke the sanctity of the CF-type restriction $B+\bar{B}+i(\bar{C} \dot{C}-\dot{\bar{C}} C)=0$. It goes without saying that the above cited requirements of the offshell nilpotency and absolute anticommutativity properties are very sacrosanct within the framework of the BRST approach to gauge and/or reparameterization invariant theories.

Against the backdrop of the above discussions, we set out to deduce the (anti-)BRST symmetry transformations $s_{a b} S=\bar{C} \dot{S}$ and $s_{b} S=C \dot{S}$ (with $S=x, p_{x}, t, p_{t}$ ) and the CF- 
type restrictions $B+\bar{B}+i(\bar{C} \dot{C}-\dot{\bar{C}} C)=0$ within the framework of MBTSA. Towards this end in our mind, first of all, we generalize the classical function $g(\tau)$ (in $\tau \longrightarrow \tau^{\prime}=g(\tau)$ $\equiv \tau-\varepsilon(\tau))$ onto a $(1,2)$-dimensional supermanifold as

$$
g(\tau) \longrightarrow \tilde{g}(\tau, \theta, \bar{\theta})=\tau-\theta \bar{C}(\tau)-\bar{\theta} C(\tau)+\theta \bar{\theta} k(\tau),
$$

where $(\bar{C}) C$ variables are the (anti)ghost variables of Equation (6) and $k(\tau)$ is a secondary variable that has to be determined from the consistency conditions (that include the off-shell nilpotency as well as absolute anticommutativity requirements). It will be noted that, due to the mappings $\left.s_{b} \leftrightarrow \partial_{\bar{\theta}}\right|_{\theta=0}$ and $\left.s_{a b} \leftrightarrow \partial_{\theta}\right|_{\bar{\theta}=0}$ [14-16], we have taken the coefficients of $\theta$ and $\bar{\theta}$ in Equation (16) as the (anti)ghost variables $(\bar{C}) C$. This has been done due to our observation in the infinitesimal reparameterization symmetry transformation $\left(\delta_{\mathrm{r}}\right)$ (where $\delta_{\mathrm{r}}$ $\tau=-\varepsilon(\tau)$ ) at the classical level. Following the basic tenet of BRST formalism, the infinitesimal parameter $\varepsilon(\tau)$ has been replaced (in the BRST-quantized theory) by the (anti)ghost variables, thereby leading to the (anti-)BRST symmetry transformations $s_{a b} \tau=-\bar{C}$ and $s_{b} \tau=-C$.

For our present 1D diffeomorphism (i.e., reparameterization) invariant theory, the generic variable $S(\tau)$ can be generalized to a supervariable $[\tilde{S}(\tilde{g}(\tau, \theta, \bar{\theta}), \theta, \bar{\theta})]$ on the $(1,2)$ -dimensional supermanifold [22] with the following super expansion along all the Grassmannian directions of the (1, 2)-dimensional supermanifold, namely,

$$
\begin{aligned}
& \tilde{S}[\tilde{\mathfrak{g}}(\tau, \theta, \bar{\theta}), \theta, \bar{\theta})]=S[\tilde{\mathfrak{g}}(\tau, \theta, \bar{\theta})]+\theta \bar{R}[\tilde{\mathfrak{g}}(\tau, \theta, \bar{\theta})] \\
& \quad+\bar{\theta} R[\tilde{\mathfrak{g}}(\tau, \theta, \bar{\theta})]+\theta \bar{\theta} Q[\tilde{\mathfrak{g}}(\tau, \theta, \bar{\theta})],
\end{aligned}
$$

where the expression for $\tilde{g}(\tau, \theta, \bar{\theta})$ is given in Equation (16). It should be noted that all the primary as well as secondary supervariables on the r.h.s. of (17) are a function of the (1, 2)-dimensional super infinitesimal diffeomorphism transformation (16). At this stage, we can perform the Taylor expansions for all the supervariables as

$$
\begin{gathered}
\theta \bar{\theta} Q(\tau-\theta \bar{C}-\bar{\theta} C+\theta \bar{\theta} k)=\theta \bar{\theta} Q(\tau), \\
\bar{\theta} R(\tau-\theta \bar{C}-\bar{\theta} C+\theta \bar{\theta} k)=\bar{\theta} R(\tau)+\theta \bar{\theta} \bar{C}(\tau) \dot{R}(\tau), \\
\theta \bar{R}(\tau-\theta \bar{C}-\bar{\theta} C+\theta \bar{\theta} k)=\theta \bar{R}(\tau)-\theta \bar{\theta} C(\tau) \dot{\bar{R}}(\tau), \\
S(\tau-\theta \bar{C}-\bar{\theta} C+\theta \bar{\theta} k)=S(\tau)-\theta \bar{C}(\tau) \dot{S}(\tau)-\bar{\theta} C(\tau) \dot{S}(\tau) \\
+\theta \bar{\theta}[k(\tau) \dot{S}(\tau)-\bar{C}(\tau) C(\tau) \ddot{S}(\tau)] .
\end{gathered}
$$

Collecting all these terms and substituting them into (17), we obtain the following super expansion for the supervariable on the $(1,2)$-dimensional supermanifold, namely,

$$
\begin{aligned}
\tilde{S}[\tilde{g}(\tau, \theta, \bar{\theta}), \theta, \bar{\theta}]= & S(\tau)+\theta(\bar{R}-\bar{C} \dot{S})+\bar{\theta}(R-C \dot{S}) \\
& +\theta \bar{\theta}[Q+\bar{C} \dot{R}-C \dot{\bar{R}}+k \dot{S}+C \bar{C} \ddot{S}]
\end{aligned}
$$

We now exploit the horizontality condition (HC) which physically implies that all the scalar variables should not transform at all under any kind of spacetime, internal, supersymmetric, etc., transformations. With respect to the $1 \mathrm{D}$ space of the trajectory of the particle, all the supervariables on the l.h.s. and r.h.s. of Equation (18) are scalars. The HC, in our case, is

$$
\tilde{S}[\tilde{g}(\tau, \theta, \bar{\theta}), \theta, \bar{\theta})]=S(\tau) .
$$

The above equality implies that all the coefficients of $\theta, \bar{\theta}$, and $\theta \bar{\theta}$ of Equation (19) should be set equal to zero. In other words, we have the following:

$$
\begin{aligned}
R & =C \dot{S}, \\
\bar{R} & =\bar{C} \dot{S}, \\
Q & =C \dot{\bar{R}}-\bar{C} \dot{R}-k \dot{S}+\bar{C} C \ddot{S} .
\end{aligned}
$$

Substitutions of the values of $R$ and $\bar{R}$ into the expression for $Q$ lead to the following:

$$
Q=-(\bar{C} \dot{C}+\dot{\bar{C}} C) \dot{S}-k \dot{S}-\bar{C} C \ddot{S}
$$

As explained before Equation (20) (i.e., exploiting the key properties of scalars), it is evident that (17) can be finally written with $\tilde{S}[\tilde{g}(\tau, \theta, \bar{\theta}), \theta, \bar{\theta}]=\tilde{S}(\tau, \theta, \bar{\theta})$ as

$$
\begin{aligned}
\tilde{S}[\tau, \theta, \bar{\theta})] & =S(\tau)+\theta \bar{R}(\tau)+\bar{\theta} R(\tau)+\theta \bar{\theta} Q(\tau) \\
& \equiv S(\tau)+\theta\left(s_{a b} S\right)+\bar{\theta}\left(s_{b} S\right)+\theta \bar{\theta}\left(s_{b} s_{a b} S\right)
\end{aligned}
$$

where, due to the well-known mappings $\left.s_{b} \leftrightarrow \partial_{\bar{\theta}}\right|_{\theta=0}$ and $\left.s_{a b} \leftrightarrow \partial_{\theta}\right|_{\bar{\theta}=0}$ [14-16], the coefficients of $\theta$ and $\bar{\theta}$ are the antiBRST and BRST symmetry transformations (cf. Equation (6)). We point out that the key properties of scalars on the r.h.s. of Equation (17) imply that we have $S[\tilde{g}(\tau, \theta, \bar{\theta})]=S$ $(\tau), R[\tilde{g}(\tau, \theta, \bar{\theta})]=R(\tau), \bar{R}[\tilde{g}(\tau, \theta, \bar{\theta})]=\bar{R}(\tau)$, and $Q[\tilde{g}(\tau, \theta$, $\bar{\theta})]=Q(\tau)$.

A comparison between (21) and (23) implies that we have already derived the nilpotent (anti-)BRST symmetry transformations $R=s_{b} S=C \dot{S}$ and $\bar{R}=s_{a b} S=\bar{C} \dot{S}$. In other words, we have obtained $s_{b} x=C \dot{x}, s_{b} p_{x}=C \dot{p}_{x}, s_{b} t=C \dot{t}, s_{b} p_{t}=C \dot{p}_{t}$, $s_{a b} x=\bar{C} \dot{x}, s_{a b} p_{x}=\bar{C} \dot{p}_{x}, s_{a b} t=\bar{C} \dot{t}$, and $s_{a b} p_{t}=\bar{C} \dot{p}_{t}$. Furthermore, it is evident that

$$
s_{b} s_{a b} S=Q=-(\bar{C} \dot{C}+\dot{\bar{C}} C) \dot{S}-k \dot{S}-\bar{C} C \ddot{S} .
$$


The requirement of the absolute anticommutativity $\left\{s_{b}\right.$, $\left.s_{a b}\right\} S=0$ implies that $s_{b} s_{a b} S=-s_{a b} s_{b} S$ which, in turn, leads to the following relationships:

$$
\begin{gathered}
s_{b} s_{a b} S=s_{b} \bar{R}=Q \equiv-(\bar{C} \dot{C}+\dot{\bar{C}} C) \dot{S}-k \dot{S}-\bar{C} C \ddot{S} \\
-s_{a b} s_{b} S=-s_{a b} R=Q \equiv-(\bar{C} \dot{C}+\dot{\bar{C}} C) \dot{S}-k \dot{S}-\bar{C} C \ddot{S}
\end{gathered}
$$

The explicit computations of the following, using the (anti-)BRST symmetry transformations of the phase variables in Equations (6) and (14), are

$$
\begin{gathered}
s_{b} \bar{R}=i B \dot{S}-\bar{C} \dot{C} \dot{S}-\bar{C} C \ddot{S} \equiv Q, \\
-s_{a b} R=-i \bar{B} \dot{S}-\dot{\bar{C}} C \dot{S}-\bar{C} C \ddot{S} \equiv Q .
\end{gathered}
$$

Equating (25) and (26), we obtain the following interesting relationship:

$$
k=-\dot{\bar{C}} C-i B \equiv i \bar{B}-\bar{C} \dot{C} \Rightarrow B+\bar{B}+i(\bar{C} \dot{C}-\dot{\bar{C}} C)=0
$$

In other words, it is the consistency conditions of the BRST formalism that lead to the determination of $k(\tau)$ in Equation (16) within the ambit of MBTSA. A close look at Equations (25)-(27) establishes that a precise determination of $Q(\tau)$ in (23) leads to (i) the validity of the absolute anticommutativity (i.e., $\left\{s_{b}, s_{a b}\right\} S=0$ ) of the off-shell nilpotent (anti-)BRST symmetries and (ii) the deduction of the (anti-)BRST invariant (This statement is true only when the whole theory is considered on a submanifold of the Hilbert space of the quantum variables where the CF-type restriction $B+\bar{B}+i(\bar{C} \dot{C}-\dot{\bar{C}} C)=0$ is satisfied. In other words, we explicitly compute $s_{b}[B+\bar{B}+i(\bar{C} \dot{C}-\dot{\bar{C}} C)]=(d / d \tau)[B+\bar{B}+i$ $(\bar{C} \dot{C}-\dot{\bar{C}} C)] C-[B+\bar{B}+i(\bar{C} \dot{C}-\dot{\bar{C}} C)] \dot{C}$ and $s_{a b}[B+\bar{B}+i(\bar{C} \dot{C}$ $-\dot{\bar{C}} C)]=(d / d \tau)[B+\bar{B}+i(\bar{C} \dot{C}-\dot{\bar{C}} C)] \bar{C}-[B+\bar{B}+i(\bar{C} \dot{C}-\dot{\bar{C}}$ $C)] \dot{\bar{C}}$ which imply that $s_{(a) b}[B+\bar{B}+i(\bar{C} \dot{C}-\dot{\bar{C}} C)]=0$ is true only on the abovementioned submanifold.) CF-type restriction $B+\bar{B}+i(\bar{C} \dot{C}-\dot{\bar{C}} C)=0$ on our theory. This statement (i.e., the (anti-)BRST invariance) is true only when the whole theory is considered on a submanifold of the Hilbert space of the quantum variables where the CF-type restriction $B+\bar{B}+i(\bar{C} \dot{C}-\dot{C} C)=0$ is satisfied. In other words, we explicitly compute $s_{b}[B+\bar{B}+i(\bar{C} \dot{C}-\dot{\bar{C}} C)]=(d / d \tau)[B+$ $\bar{B}+i(\bar{C} \dot{C}-\dot{\bar{C}} C)] C-[B+\bar{B}+i(\bar{C} \dot{C}-\dot{\bar{C}} C)] \dot{C}$ and $s_{b}[B+\bar{B}+i$ $(\bar{C} \dot{C}-\dot{\bar{C}} C)]=(d / d \tau)[B+\bar{B}+i(\bar{C} \dot{C}-\dot{\bar{C}} C)] \bar{C}-[B+\bar{B}+i(\bar{C} \dot{C}$ $-\dot{\bar{C}} C)] \dot{\bar{C}}$ which imply that $s_{(a) b}[B+\bar{B}+i(\bar{C} \dot{C}-\dot{\bar{C}} C)]=0$ is true only on the abovementioned submanifold.

We conclude this section with the following useful and crucial remarks. First, we set out to derive the (anti-)BRST symmetry transformations (corresponding to the classical reparameterization symmetry transformations) for the phase variables (cf. Equation (6)). We have accomplished this goal in Equation (21). Second, we have derived the CF-type restriction $B+\bar{B}+i(\bar{C} \dot{C}-\dot{\bar{C}} C)=0$ within the purview of
MBTSA (cf. Equation (27)) which is actually hidden in the determination of $Q(\tau)$ in Equation (23). Third, for the application of the theoretical potential of MBTSA, we have taken the full super expansion of the generic supervariable (cf. Equation (17)) along all the possible Grassmannian directions of the $(1,2)$-dimensional supermanifold. Fourth, unlike the application of the BT superfield/supervariable approach to the gauge theories [14-16] where spacetime does not change, in the case of MBTSA, the super diffeomorphism transformation (16) has been taken into account in all the basic as well as secondary supervariables. Fifth, taking into account the inputs from Equations (21) and (26), we obtain the following super expansion of the generic variable $S(\tau)$, namely,

$$
\begin{aligned}
\tilde{S}^{(\mathrm{h})}(\tau, \theta, \bar{\theta})= & S(\tau)+\theta(\bar{C} \dot{S})+\bar{\theta}(C \dot{S}) \\
& +\theta \bar{\theta}[i B \dot{S}-\bar{C} \dot{C} \dot{S}-\bar{C} C \ddot{S}] \equiv S(\tau)+\theta\left(s_{a b} S\right) \\
& +\bar{\theta}\left(s_{b} S\right)+\theta \bar{\theta}\left(s_{b} s_{a b} S\right),
\end{aligned}
$$

where $S=x, p_{x}, t, p_{t}$ and the superscript (h) on the supervariable $\tilde{S}(\tau, \theta, \bar{\theta})$ denotes that this supervariable has been obtained after the application of HC. Finally, the standard nilpotent (anti-)BRST symmetry transformations (8) dictate that we can have the following (anti)chiral super expansions for the supervariables corresponding to $(\bar{C}) C$, namely,

$$
\begin{aligned}
& C(\tau) \longrightarrow F^{(\mathrm{c})}(\tau, \theta)=C(\tau)+\theta(i \bar{B}) \equiv C(\tau)+\theta\left(s_{a b} C\right), \\
& \bar{C}(\tau) \longrightarrow \bar{F}^{(\mathrm{ac})}(\tau, \bar{\theta})=\bar{C}(\tau)+\bar{\theta}(i B) \equiv \bar{C}(\tau)+\bar{\theta}\left(s_{b} \bar{C}\right),
\end{aligned}
$$

where the superscripts (c) and (ac) denote the chiral and antichiral supervariables. The above observation gives us a clue that we should exploit the theoretical strength of ACSA to BRST formalism for our further discussions.

\section{Coupled Lagrangians and Quantum (Anti-)BRST Symmetries Corresponding to the Classical Reparameterization Symmetry Transformations}

In addition to the quantum (anti-)BRST symmetries in (6), (8), and (14), we derive all the other off-shell nilpotent and absolutely anticommuting (anti-)BRST symmetries corresponding to the classical infinitesimal and continuous reparameterization symmetry transformations (2). We exploit the strength of the sacrosanct requirements of off-shell nilpotency and absolute anticommutativity properties. In this context, we point out that we have already derived $s_{b} C=C \dot{C}$ and $s_{a b} \bar{C}=\bar{C} \dot{\bar{C}}$ by invoking the sanctity of the off-shell nilpotency $\left(s_{(a) b}^{2}=0\right)$ property for the phase variables (i.e., $s_{(a) b}^{2} S=0$, $\left.S=x, p_{x}, t, p_{t}\right)$. It is interesting to note that the following absolute anticommutativity requirements, namely, 


$$
\begin{aligned}
& \left\{s_{b}, s_{a b}\right\} C=0 \Rightarrow s_{b} \bar{B}=\dot{\bar{B}} C-\bar{B} \dot{C}, \\
& \left\{s_{b}, s_{a b}\right\} \bar{C}=0 \Rightarrow s_{a b} B=\dot{B} \bar{C}-B \dot{\bar{C}},
\end{aligned}
$$

lead to the derivation of the $s_{b} \bar{B}$ and $s_{a b} B$. We can readily check that $s_{b}^{2} \bar{B}=0$ and $s_{a b}^{2} B=0$ are satisfied due to our knowledge of the BRST and anti-BRST symmetry transformations $s_{b} C=C \dot{C}$ and $s_{a b} \bar{C}=\bar{C} \dot{\bar{C}}$ and the fermionic $\left(C^{2}\right.$ $=\bar{C}^{2}=0, C \bar{C}+\bar{C} C=0$ ) nature of the (anti)ghost variables $(\bar{C}) C$. We further note that $\left\{s_{b}, s_{a b}\right\} B=0$ and $\left\{s_{b}, s_{a b}\right\} \bar{B}$ $=0$. The requirement of the absolute anticommutativity on the $E(\tau)$ variable leads to

$$
\left\{s_{b}, s_{a b}\right\} E(\tau)=\frac{d}{d \tau}[i\{B+\bar{B}+i(\bar{C} \dot{C}-\dot{\bar{C}} C)\} E(\tau)] .
$$

Thus, we emphasize that the absolute anticommutativity property $\left(s_{b} s_{a b}+s_{a b} s_{b}=0\right)$ on the phase variables (cf. Equation (15)) as well as on the Lagrange multiplier variable (cf. Equation (31)) is satisfied if and only if the CF-type restriction is invoked. In the full blaze of glory, the quantum (anti-)BRST symmetry transformations (corresponding to the infinitesimal reparameterization symmetry transformations (2)) are as follows:

$$
\begin{aligned}
s_{a b} x & =\bar{C} \dot{x}, \\
s_{a b} p_{x} & =\bar{C} \dot{p}_{x}, \\
s_{a b} t & =\bar{C} \dot{t}, \\
s_{a b} p_{t} & =\bar{C} \dot{p}_{t}, \\
s_{a b} E & =\frac{d}{d \tau}(\bar{C} E), \\
s_{a b} C & =i \bar{B}, \\
s_{a b} \bar{C} & =\bar{C} \dot{\bar{C}}, \\
s_{a b} \bar{B} & =0, \\
s_{a b} B & =\dot{B} \bar{C}-B \overline{\bar{C}}, \\
s_{b} x & =C \dot{x}, \\
s_{b} p_{x} & =C \dot{\bar{p}} \\
s_{b} t & =C \dot{t}, \\
s_{b} p_{t} & =C \dot{\bar{p}}, \\
s_{b} E & =\frac{d}{d \tau}(C E), \\
s_{b} \bar{C} & =i B \\
s_{b} C & =C \dot{C} \\
s_{b} B & =0, \\
s_{b} \bar{B} & =\dot{\bar{B}} C-\bar{B} \dot{C} . \\
&
\end{aligned}
$$

The above fermionic symmetry transformations are offshell nilpotent and absolutely anticommuting provided that the whole theory is considered on a submanifold of the space of quantum variables where the CF-type restriction $B+\bar{B}+i(\bar{C} \dot{C}-\dot{\bar{C}} C)=0$ is satisfied.

The existence of the above CF-type restriction leads to the derivation of the coupled (but equivalent) Lagrangians (i.e., $L_{B}$ and $L_{\bar{B}}$ ) as follows:

$$
\begin{aligned}
& L_{B}=L_{\mathrm{f}}+s_{b} s_{a b}\left[\frac{i E^{2}}{2}-\frac{\bar{C} C}{2}\right], \\
& L_{\bar{B}}=L_{\mathrm{f}}-s_{a b} s_{b}\left[\frac{i E^{2}}{2}-\frac{\bar{C} C}{2}\right] .
\end{aligned}
$$

We point out that the terms inside the square brackets are the same as in Equation (10) for the BRST analysis of the classical gauge symmetry transformations (4). Furthermore, in contrast to the unique (anti-)BRST invariant Lagrangian (cf. Equation (11)) (corresponding to the classical gauge symmetry transformations), we have obtained here a set of coupled (but equivalent) (anti-)BRST invariant Lagrangians in Equation (34). This has happened because of the fact that the CF-type restriction $(b+\bar{b}=0)$ is trivial in the case of the former while it is a nontrivial restriction $[B+\bar{B}+i(\bar{C} \dot{C}-\dot{\bar{C}} C)$ $=0$ ] in the context of the latter.

One can readily compute the operation of $s_{(a) b}$ on the quantities in the square brackets of Equation (34). In the full blaze of their glory, the coupled (but equivalent) Lagrangians $L_{B}$ and $L_{\bar{B}}$ are as follows (It will be worthwhile to mention here that the form of the gauge-fixing and Faddeev-Popov ghost terms is the same as in the cases of NSUSY (i.e., scalar) and SUSY (i.e., spinning) relativistic particles [23, 24].):

$$
\begin{aligned}
& L_{B}=L_{\mathrm{f}}+B[E \dot{E}-i(2 \dot{\bar{C}} C+\bar{C} \dot{C})]+\frac{B^{2}}{2}-i E \dot{E} \dot{\bar{C}} C-i E^{2} \dot{\bar{C}} \dot{C}-\dot{\bar{C}} \bar{C} \dot{C} C \\
& L_{\bar{B}}=L_{\mathrm{f}}-\bar{B}[E \dot{E}-i(2 \bar{C} \dot{C}+\dot{\bar{C}} C)]+\frac{\bar{B}^{2}}{2}-i E \dot{E} \bar{C} \dot{C}-i E^{2} \dot{\bar{C}} \dot{C}-\dot{\bar{C}} \bar{C} \dot{C} C
\end{aligned}
$$

where the subscripts $B$ and $\bar{B}$ on the Lagrangians are appropriate because $L_{B}$ depends uniquely on the NakanishiLautrup auxiliary variable $B$ (where $\bar{B}$ is not present at all). Similarly, the Lagrangian $L_{\bar{B}}$ is uniquely dependent on $\bar{B}$. They are coupled because the EL-EOMs with respect to $B$ and $\bar{B}$ from $L_{B}$ and $L_{\bar{B}}$, respectively, yield

$$
\begin{aligned}
& B=-E \dot{E}+2 i \dot{\bar{C}} C+i \bar{C} \dot{C} \\
& \bar{B}=E \dot{E}-2 i \bar{C} \dot{C}-i \dot{\bar{C}} C
\end{aligned}
$$

which lead to the deduction of the CF-type restrictions $B+$ $\bar{B}+i(\bar{C} \dot{C}-\dot{\bar{C}} C)=0$. Furthermore, the condition $L_{B} \equiv L_{\bar{B}}$ also demonstrates the existence of the CF-type restriction $B+\bar{B}$ $+i(\bar{C} \dot{C}-\dot{\bar{C}} C)=0$ on our theory (cf. Appendix A below). It will be worthwhile to mention here that the form of the gauge-fixing and Faddeev-Popov ghost terms in Equation 
(35) is the same as in the cases of NSUSY (i.e., scalar) and SUSY (i.e., spinning) relativistic particles [23, 24].

At this stage, we are in the position to study the (anti-)BRST symmetries of the Lagrangians $L_{B}$ and $L_{\bar{B}}$. It is straightforward to note that we have the following:

$$
\begin{gathered}
s_{b} L_{B}=\frac{d}{d \tau}\left[C L_{\mathrm{f}}+B^{2} C-i B \bar{C} \dot{C} C+E \dot{E} B C+E^{2} B \dot{C}\right], \\
s_{a b} L_{\bar{B}}=\frac{d}{d \tau}\left[\bar{C} L_{\mathrm{f}}+\bar{B}^{2} \bar{C}-i \bar{B} \dot{\bar{C}} \bar{C} C-E \dot{E} \bar{B} \bar{C}-E^{2} \bar{B} \dot{\bar{C}}\right] .
\end{gathered}
$$

The above observations demonstrate that the action integrals $S_{1}=\int_{-\infty}^{\infty} d \tau L_{B}$ and $S_{2}=\int_{-\infty}^{\infty} d \tau L_{\bar{B}}$ remain invariant under the SUSY-type (i.e., fermionic) off-shell nilpotent, continuous, and infinitesimal (anti-)BRST symmetry transformations for the physical variables that vanish off at $\tau= \pm \infty$. At this crucial juncture, we establish the equivalence of the coupled Lagrangians $L_{B}$ and $L_{\bar{B}}$ w.r.t. the (anti-)BRST symmetry transformations $\left[s_{(a) b}\right]$. In this context, we apply $s_{a b}$ on $L_{B}$ and $s_{b}$ on $L_{\bar{B}}$ to obtain the following:

$$
\begin{aligned}
s_{a b} L_{B}= & \frac{d}{d \tau}\left[\bar{C} L_{\mathrm{f}}+E \dot{E}(i \dot{\bar{C}} \bar{C} C+B \bar{C})+E^{2}(i \dot{\bar{C}} \bar{C} \dot{C}+B \dot{\bar{C}})\right. \\
& \left.+B^{2} \bar{C}+i(2 B-\bar{B}) \dot{\bar{C}} \bar{C} C\right]+[B+\bar{B}+i(\bar{C} \dot{C}-\dot{\bar{C}} C)] \\
& \cdot(2 i \dot{\bar{C}} \bar{C} \dot{C}-2 B \dot{\bar{C}}-E \dot{\bar{E}} \dot{\bar{C}}+i \ddot{\bar{C}} \bar{C} C) \\
& -\frac{d}{d \tau}[B+\bar{B}+i(\bar{C} \dot{C}-\dot{\bar{C}} C)]\left[B \bar{C}+E^{2} \dot{\bar{C}}\right] \\
s_{b} L_{\bar{B}}= & \frac{d}{d \tau}\left[C L_{\mathrm{f}}+E \dot{E}(i \bar{C} C \dot{C}-\bar{B} C)+E^{2}(i \dot{\bar{C}} C \dot{C}-\bar{B} \dot{C})\right. \\
& \left.+\bar{B}^{2} C-i(2 \bar{B}-B) \bar{C} C \dot{C}\right]+[B+\bar{B}+i(\bar{C} \dot{C}-\dot{\bar{C}} C)] \\
& \cdot(-2 i \dot{\bar{C}} C \dot{C}-2 \bar{B} \dot{C}+E \dot{E} \dot{C}+i \bar{C} \ddot{C} C) \\
& +\frac{d}{d \tau}[B+\bar{B}+i(\bar{C} \dot{C}-\dot{\bar{C}} C)]\left[+E^{2} \dot{C}-\bar{B} C\right]
\end{aligned}
$$

which demonstrate that the coupled Lagrangians $L_{B}$ and $L_{\bar{B}}$ (and corresponding action integrals) respect both (i.e., BRST and anti-BRST) symmetry transformations together provided that the whole theory is considered on a supermanifold in the Hilbert space of quantum variables where the CF-type restriction $B+\bar{B}+i(\bar{C} \dot{C}-\dot{\bar{C}} C)=$ 0 is satisfied. It should be recalled that, under the latter restriction, we also have the absolute anticommutativity property (i.e., $\left\{s_{b}, s_{a b}\right\}=0$ ) of the (anti-)BRST symmetry transformations.

We end this section with the following key comments. First, the properties of the off-shell nilpotency and absolute anticommutativity are sacrosanct in the realm of the BRST approach to gauge and/or diffeomorphism invariant theories.
Second, physically, the first property (i.e., off-shell nilpotency) implies that these fermionic symmetry transformations are of supersymmetric type as they transform bosonic variables to fermionic variables and vice versa. Third, the property of the absolute anticommutativity encodes the linear independence of the BRST and anti-BRST symmetry transformations. Fourth, the absolute anticommutativity property owes its origin to the existence of the CF-type restrictions which are connected with the concepts of gerbes $[7,8]$. Fifth, as the classical gauge theory is characterized by the first-class constraints, in an exactly similar fashion, the quantum gauge and/or diffeomorphism (i.e., (anti-)BRST) invariant theories are characterized by the existence of the CF-type restrictions within the ambit of BRST formalism. Sixth, the coupled Lagrangians $L_{B}$ and $L_{\bar{B}}$ are equivalent because both of them respect BRST and anti-BRST symmetry transformations as is clear from Equations (37)-(40) provided that the whole theory is considered on the submanifold of the total Hilbert space of the quantum variables where the CF-type restriction $B+\bar{B}+i(\bar{C} \dot{C}-\dot{\bar{C}} C)=0$ is satisfied.

\section{Quantum Off-Shell Nilpotent (Anti-)BRST Symmetries of the Other Variables: ACSA}

In this section, we derive the nilpotent (anti-)BRST symmetry transformations $\left[s_{(a) b}\right]$ for all the other variables (cf. Equations (32) and (33)) besides the phase space variables $\left(x, p_{x}, t\right.$, and $\left.p_{t}\right)$ whose (anti-)BRST symmetries have already been derived in Section 3 by exploiting the theoretical potential of MBTSA. To achieve the above goal, we exploit the ideas behind ACSA to BRST formalism [25-29]. In this context, first of all, we focus on the derivation of the BRST symmetry transformations $s_{b} B=0, s_{b}$ $\bar{B}=\dot{\bar{B}} C-\bar{B} \dot{C}, s_{b} C=C \dot{C}$, and $s_{b} E=\dot{E} C+E \dot{C}$ (cf. Equation (33)). For this purpose, we generalize the ordinary variables $(B(\tau), \bar{B}(\tau), C(\tau)$, and $E(\tau))$ onto a $(1,1)$-dimensional antichiral super submanifold as follows:

$$
\begin{aligned}
& B(\tau) \longrightarrow B(\tau, \bar{\theta})=B(\tau)+\bar{\theta} f_{1}(\tau), \\
& \bar{B}(\tau) \longrightarrow \bar{B}(\tau, \bar{\theta})=\bar{B}(\tau)+\bar{\theta} f_{2}(\tau), \\
& C(\tau) \longrightarrow F(\tau, \bar{\theta})=C(\tau)+\bar{\theta} b_{1}(\tau), \\
& E(\tau) \longrightarrow \Sigma(\tau, \bar{\theta})=E(\tau)+\bar{\theta} f_{3}(\tau),
\end{aligned}
$$

where we note that $f_{1}, f_{2}$, and $f_{3}$ are the fermionic secondary variables and $b_{1}(\tau)$ is the bosonic secondary variable because of the fermionic $\left(\bar{\theta}^{2}=0\right)$ nature of the Grassmannian variable $\bar{\theta}$ which characterizes the antichiral super submanifold (along with the bosonic evolution parameter $\tau)$. It is elementary to note that the observation $s_{b} B=0$ implies the following super expansion (in view of the fact that $\left.\partial_{\bar{\theta}} \leftrightarrow s_{b}\right)$, namely, 


$$
B^{(\mathrm{b})}(\tau, \bar{\theta})=B(\tau)+\bar{\theta}(0) \equiv B(\tau)+\bar{\theta}\left(s_{b} B\right),
$$

where the superscript (b) on the antichiral supervariable $B(\tau, \bar{\theta})$ denotes that the coefficient of $\bar{\theta}$ yields the BRST symmetry transformation $s_{b} B=0$ due to the trivial equality $B(\tau, \bar{\theta})=B(\tau)$ which emerges from the observation that the Nakanishi-Lautrup auxiliary variable $B(\tau)$ is a BRST invariant quantity (cf. Equation (33)). In other words, we have found out that the secondary variable $f_{1}(\tau)=0$ in the super expansions (41).

At this stage, we find other nontrivial BRST invariant quantities for the derivation of the secondary variables $b_{1}$, $f_{2}$, and $f_{3}$ of Equation (41). We observe that (We have specifically taken here $s_{b}(C \dot{x})=0$ for our purpose. However, one can take the general expression $s_{b} S=C \dot{S}\left(S=x, p_{x}, t, p_{t}\right)$ for the derivation of $b_{1}(\tau)=C \dot{C}$.) $s_{b}(C \dot{x})=0, s_{b}[\dot{\bar{B}} C-\bar{B} \dot{C}]=0$, and $s_{b}[E \dot{C}+\dot{E} C]=0$. We have specifically taken here $s_{b}(C \dot{x})$ $=0$ for our purpose. However, one can take the general expression $s_{b} S=C \dot{C}\left(S=x, p_{x}, t, p_{t}\right)$ for the derivation of $b_{1}(\tau)=C \dot{C}$. The basic tenet of the ACSA to BRST formalism requires that the quantities in the square brackets have to be independent of the Grassmannian variable $\bar{\theta}$ when they are generalized onto a $(1,1)$-dimensional super submanifold, namely,

$$
\begin{gathered}
F(\tau, \bar{\theta}) \dot{X}^{(\mathrm{h}, \mathrm{ac})}(\tau, \bar{\theta})=C(\tau) \dot{x}(\tau), \\
B(\tau, \bar{\theta}) F(\tau, \bar{\theta})-B(\tau, \bar{\theta}) \dot{F}(\tau, \bar{\theta})=\dot{\bar{B}}(\tau) C(\tau)-\bar{B}(\tau) \dot{C}(\tau), \\
\Sigma(\tau, \bar{\theta}) \dot{F}(\tau, \bar{\theta})+\dot{\Sigma}(\tau, \bar{\theta}) F(\tau, \bar{\theta})=E(\tau) \dot{C}(\tau)+\dot{E}(\tau) C(\tau),
\end{gathered}
$$

where $X^{(\mathrm{h}, \mathrm{ac})}$ is the antichiral limit of the full expansion of $X^{(\mathrm{h})}(\tau, \theta, \bar{\theta})$ obtained after the application of HC (cf. Equation (28)), namely,

$$
X^{(\mathrm{h})}(\tau, \theta, \bar{\theta})=x(\tau)+\theta(\bar{C} \dot{x})+\bar{\theta}(C \dot{x})+\theta \bar{\theta}[i B \dot{x}-\bar{C} \dot{C} \dot{x}-\bar{C} C \ddot{x}],
$$

which has been obtained (cf. Equation (28)) in Section 3 using the theoretical strength of MBTSA. In other words, from the top entry of Equation (43), we have the following restriction:

$$
\begin{gathered}
F(\tau, \bar{\theta}) \dot{X}^{(\mathrm{h}, \mathrm{ac})}(\tau, \bar{\theta})=C(\tau) \dot{x}(\tau) \Rightarrow\left[C(\tau)+\bar{\theta} b_{1}(\tau)\right] \\
\cdot[\dot{x}+\bar{\theta}(\dot{C} \dot{x}+C \ddot{x})]=C(\tau) \dot{x}(\tau) .
\end{gathered}
$$

From the above relationship, we obtain $b_{1}(\tau)=C \dot{C}$. Thus, we have the following:

$$
F^{(\mathrm{b})}(\tau, \bar{\theta})=C(\tau)+\bar{\theta}(C \dot{C}) \equiv C(\tau)+\bar{\theta}\left(s_{b} C\right),
$$

where the superscript (b) on the l.h.s. of the supervariable denotes that the coefficient of $\bar{\theta}$ is nothing but the BRST sym- metry transformation $s_{b} C$. We have to use the above super expansion in the second entry from the top in (43) to obtain the following:

$$
B(\tau, \bar{\theta}) F^{(\mathbf{b})}(\tau, \bar{\theta})-B(\tau, \bar{\theta}) \dot{F}^{(\mathrm{b})}(\tau, \bar{\theta})=\dot{\bar{B}}(\tau) C(\tau)-\bar{B}(\tau) \dot{C}(\tau)
$$

In other words, we have the following equality:

$$
\begin{gathered}
{\left[\dot{\bar{B}}+\bar{\theta} \dot{f}_{2}(\tau)\right][C(\tau)+\bar{\theta}(C \dot{C})]-\left[\bar{B}(\tau)+\bar{\theta} f_{2}(\tau)\right]} \\
\cdot[\dot{C}(\tau)+\bar{\theta}(C \ddot{C})]=\dot{\bar{B}}(\tau) C(\tau)-\bar{B}(\tau) \dot{C}(\tau),
\end{gathered}
$$

which yields the following condition on the secondary variable $f_{2}$, namely,

$$
\dot{f}_{2} C-f_{2} \dot{C}-\dot{\bar{B}} \dot{C} C+\bar{B} \ddot{C} C=0 .
$$

It is straightforward to note that $f_{2}=\dot{\bar{B}} C-\bar{B} \dot{C}$ satisfies the above condition in a precise manner. We point out that the last entry (from the top) of Equation (43) can be rewritten, in view of our the super expansion in Equation (46), as follows:

$$
\Sigma(\tau, \bar{\theta}) \dot{F}^{(\mathrm{b})}(\tau, \bar{\theta})+\dot{\Sigma}(\tau, \bar{\theta}) F^{(\mathrm{b})}(\tau, \bar{\theta})=E(\tau) \dot{C}(\tau)+\dot{E}(\tau) C(\tau)
$$

The substitutions of expansions from (41) and (46) lead to the following condition on the secondary variable $f_{3}(\tau)$ (present in the expansion of $\Sigma(\tau, \bar{\theta})$ ), namely,

$$
f_{3} \dot{C}+\dot{f}_{3} C-E \ddot{C} C-\dot{E} \dot{C} C=0
$$

which is satisfied by the choice $f_{3}=E \dot{C}+\dot{E} C$. Hence, we have the following super expansions (with the BRST symmetry transformations (33) as input), namely,

$$
\begin{aligned}
& B^{(\mathrm{b})}(\tau, \bar{\theta})=\bar{B}(\tau)+\bar{\theta}(\dot{\bar{B}} C-\bar{B} \dot{C}) \equiv \bar{B}(\tau)+\bar{\theta}\left(s_{b} \bar{B}\right), \\
& \Sigma^{(\mathrm{b})}(\tau, \bar{\theta})=E(\tau)+\bar{\theta}(E \dot{C}+\dot{E} C) \equiv E(\tau)+\bar{\theta}\left(s_{b} E\right)
\end{aligned}
$$

where the coefficients of $\bar{\theta}$ (in view of $\partial_{\bar{\theta}} \leftrightarrow s_{b}$ ) are the BRST symmetry transformations (33). For the convenience of the readers, we have performed the explicit computations of $f_{3}=E \dot{C}+\dot{E} C$ and $f_{2}=\dot{\bar{B}} C-\bar{B} \dot{C}$ in Appendix B. It is clear that we have already computed the BRST transformations $s_{b} B=0, s_{b} C=C \dot{C}, s_{b} \bar{B}=\dot{\bar{B}} C-\bar{B} \dot{C}$, and $s_{b} E=E \dot{C}+$ $\dot{E} C$ by exploiting the virtues of ACSA in Equations (42), (46), and (52).

We concentrate now on the derivation of the anti-BRST symmetry transformations (32) by exploiting the theoretical strength of ACSA to BRST formalism. It is obvious that, in Section 3 , we have already computed $s_{a b} S=\bar{C} \dot{S}\left(S=x, p_{x}, t\right.$, 
$\left.p_{t}\right)$ and $s_{a b} C=i \bar{B}$ by exploiting MBTSA to BRST formalism. Our objective in the present part of our section is to derive $s_{a b} \bar{B}=0, s_{a b} \bar{C}=\bar{C} \dot{\bar{C}}, s_{a b} B=\dot{B} \bar{C}-B \dot{\bar{C}}$, and $s_{a b} E=E \dot{\bar{C}}+\dot{E} \bar{C}$ by exploiting ACSA to BRST formalism. In this context, first of all, we generalize the ordinary variables onto a $(1,1)$ -dimensional chiral super submanifold as

$$
\begin{aligned}
& \bar{B}(\tau) \longrightarrow B(\tau, \theta)=\bar{B}(\tau)+\theta \bar{f}_{1}(\tau), \\
& B(\tau) \longrightarrow B(\tau, \theta)=B(\tau)+\theta \bar{f}_{2}(\tau), \\
& \bar{C}(\tau) \longrightarrow \bar{F}(\tau, \theta)=\bar{C}(\tau)+\theta \bar{b}_{1}(\tau), \\
& E(\tau) \longrightarrow \Sigma(\tau, \theta)=E(\tau)+\theta \bar{f}_{3}(\tau),
\end{aligned}
$$

where $f_{1}, f_{2}$, and $f_{3}$ are the fermionic secondary variables, $\bar{b}_{1}(\tau)$ is a bosonic secondary variable, and the above $(1,1)$ -dimensional chiral super submanifold is parameterized by $(\tau, \theta)$. It is straightforward to note that $s_{a b} \bar{B}=0$ implies that $B(\tau, \theta)=B(\tau)$, and as a consequence, we have $\bar{f}_{1}(\tau)=0$ which leads to

$$
B^{(\mathrm{ab})}(\tau, \theta)=\bar{B}(\tau)+\theta(0) \equiv \bar{B}(\tau)+\theta\left(s_{a b} \bar{B}\right),
$$

where the superscript $(a b)$ on the chiral supervariable denotes that we have obtained $s_{a b} \bar{B}=0$ as the coefficient of $\theta$. The other useful and interesting anti-BRST invariant quantities of our interest (cf. Equation (32)) are

$$
\begin{aligned}
s_{a b}[\dot{B} \bar{C}-B \dot{\bar{C}}] & =0, \\
s_{a b}[E \dot{\bar{C}}+\dot{E} \bar{C}] & =0, \\
s_{a b}[\bar{C} \dot{\bar{x}}] & =0 .
\end{aligned}
$$

The quantities in the square brackets can be generalized onto the $(1,1)$-dimensional chiral super submanifold. Following the fundamental requirement(s) of ACSA to BRST formalism, these quantities must be independent of the Grassmannian variable $\theta$. In other words, we have the following restrictions on the chiral supervariables:

$$
\begin{aligned}
B(\tau, \theta) \bar{F}(\tau, \theta)-B(\tau, \theta) \dot{\bar{F}}(\tau, \theta) & =\dot{B}(\tau) \bar{C}(\tau)-B(\tau) \dot{\bar{C}}(\tau), \\
\Sigma(\tau, \theta) \dot{\bar{F}}(\tau, \theta)+\dot{\Sigma}(\tau, \theta) \bar{F}(\tau, \theta) & =E(\tau) \dot{\bar{C}}(\tau)+\dot{E}(\tau) \bar{C}(\tau), \\
\bar{F}(\tau, \theta) \dot{X}^{(h, c)}(\tau, \theta) & =\bar{C}(\tau) \dot{x}(\tau),
\end{aligned}
$$

where $X^{(\mathrm{h}, \mathrm{c})}(\tau, \theta)$ is the chiral limit of the super expansion in Equation (44). In other words, we have the following explicit expression for the supervariable $X^{(\mathrm{h}, \mathrm{c})}(\tau, \theta)$, namely,

$$
X^{(\mathrm{h}, \mathrm{c})}(\tau, \theta)=x(\tau)+\theta(\bar{C} \dot{x}) .
$$

Taking the expansions from (53) and (57), we find that the last entry of Equation (56) yields $\bar{b}_{1}(\tau)=\bar{C} \dot{\bar{C}}$. Hence, we have obtained the following super expansion:

$$
\bar{F}^{(\mathrm{ab})}(\tau, \theta)=\bar{C}(\tau)+\theta(\bar{C} \dot{\bar{C}}) \equiv \bar{C}(\tau)+\theta\left(s_{a b} \bar{C}\right),
$$

where the superscript (ab) on the chiral supervariable on the 1.h.s. denotes that it has been derived after the application of the anti-BRST invariant restriction in (56). The coefficient of $\theta$ is nothing but the anti-BRST symmetry transformation $s_{a b} \bar{C}=\bar{C} \dot{\bar{C}}$. This equation also shows that $\partial_{\theta} \leftrightarrow s_{a b}$ and it leads to the anti-BRST symmetry for $\bar{C}$.

We utilize now the two top entries of (56) where we use the explicit expansion for $\bar{F}^{(\mathrm{ab})}(\tau, \theta)$ of (58) in the following restrictions on the supervariables, namely,

$$
\begin{gathered}
B(\tau, \theta) \bar{F}^{(a b)}(\tau, \theta)-B(\tau, \theta) \dot{\bar{F}}^{(a b)}(\tau, \theta)=\dot{B}(\tau) \bar{C}(\tau)-B(\tau) \dot{\bar{C}}(\tau), \\
\Sigma(\tau, \theta) \dot{\bar{F}}^{(\mathrm{ab})}(\tau, \theta)+\dot{\Sigma}(\tau, \theta) \bar{F}^{(\mathrm{ab})}(\tau, \theta)=E(\tau) \dot{\bar{C}}(\tau)+\dot{E}(\tau) \bar{C}(\tau) .
\end{gathered}
$$

The substitutions from (53) and (58) lead to

$$
\begin{aligned}
& \dot{\bar{f}}_{2} \bar{C}-\bar{f}_{2} \dot{\bar{C}}-\dot{B} \dot{\bar{C}} \bar{C}+B \ddot{\bar{C}} \bar{C}=0, \\
& \bar{f}_{3} \dot{\bar{C}}+\dot{\bar{f}}_{3} \bar{C}-E \ddot{\bar{C}} \bar{C}-E \dot{\bar{C}} \bar{C}=0 .
\end{aligned}
$$

It is straightforward, following the theoretical tricks of Appendix B, to find out the solutions for the secondary variables $\bar{f}_{2}(\tau)$ and $\bar{f}_{3}(\tau)$ which are as follows:

$$
\begin{aligned}
\bar{f}_{2}(\tau) & =\dot{B} \bar{C}-B \dot{\bar{C}}, \\
\bar{f}_{3} & =E \dot{\bar{C}}+\dot{E} \bar{C} .
\end{aligned}
$$

Substitutions of these secondary variables into the super expansions (53) lead to the determination of the anti-BRST symmetry transformations for the variables $B(\tau)$ and $E(\tau)$ as the coefficients of $\theta$ in the following:

$$
\begin{aligned}
& \Sigma^{(\mathrm{ab})}(\tau, \theta)=E(\tau)+\theta(E \dot{\bar{C}}+\dot{E} \bar{C}) \equiv E(\tau)+\theta\left[s_{a b} E(\tau)\right], \\
& B^{(\mathrm{ab})}(\tau, \theta)=B(\tau)+\theta(\dot{B} \bar{C}-B \dot{\bar{C}}) \equiv B(\tau)+\theta\left[s_{a b} B(\tau)\right],
\end{aligned}
$$

where the superscript (ab) on the chiral supervariable denotes that these supervariables have been obtained after the applications of the anti-BRST invariant restrictions (56). Moreover, the above observation establishes that $s_{a b}$ $\Leftrightarrow \partial_{\theta}$ which implies that the nilpotency $\left(s_{a b}^{2}=0, \partial_{\theta}^{2}=0\right)$ properties of $s_{a b}$ and $\partial_{\theta}$ are connected with each other. Thus, we have obtained all the anti-BRST symmetry transformations (besides the phase variables) in our Equations (54), (58), and (62). This completes our discussion on the derivation of the off-shell nilpotent and absolutely anticommuting (anti-)BRST symmetry transformations (32) and (33) within the ambit of ACSA to BRST formalism. 


\section{Symmetry Invariance of the Lagrangians: ACSA}

In this section, we establish the equivalence of the coupled Lagrangians $L_{B}$ and $L_{\bar{B}}$ as far as the (anti-)BRST symmetry invariance (within the purview of ACSA to BRST formalism) is concerned. We accomplish this objective by generalizing the ordinary Lagrangians to their counterpart super Lagrangians as

$$
\begin{aligned}
L_{\bar{B}} \longrightarrow \tilde{L}_{\overline{\bar{B}}}^{(\mathrm{c})}(\tau, \theta)=\tilde{L}_{\mathrm{f}}^{(\mathrm{c})}(\tau, \theta)-B^{(\mathrm{ab})}(\tau, \theta) & \cdot\left[\Sigma^{(\mathrm{ab})}(\tau, \theta) \dot{\Sigma}^{(\mathrm{ab})}(\tau, \theta)-i\left\{2 \bar{F}^{(\mathrm{ab})}(\tau, \theta) \dot{F}^{(\mathrm{ab})}(\tau, \theta)\right.\right. \\
& \left.\left.+\dot{\bar{F}}^{(\mathrm{ab})}(\tau, \theta) F^{(\mathrm{ab})}(\tau, \theta)\right\}\right]+\frac{1}{2} B^{(\mathrm{ab})}(\tau, \theta) B^{(\mathrm{ab})}(\tau, \theta) \\
& -i \Sigma^{(\mathrm{ab})}(\tau, \theta) \Sigma^{(\mathrm{ab})}(\tau, \theta) \dot{\bar{F}}^{(\mathrm{ab})}(\tau, \theta) \dot{F}^{(\mathrm{ab})}(\tau, \theta)-i \Sigma^{(\mathrm{ab})} \\
& \cdot(\tau, \theta) \dot{\Sigma}^{(\mathrm{ab})}(\tau, \theta) \bar{F}^{(\mathrm{ab})}(\tau, \theta) \dot{F}^{(\mathrm{ab})}(\tau, \theta)-\dot{\bar{F}}^{(\mathrm{ab})}(\tau, \theta) \bar{F}^{(\mathrm{ab})} \\
& \cdot(\tau, \theta) \dot{F}^{(\mathrm{ab})}(\tau, \theta) F^{(\mathrm{ab})}(\tau, \theta), \\
L_{B} & \longrightarrow \tilde{L}_{B}^{(\mathrm{ac})}(\tau, \bar{\theta})=\tilde{L}_{\mathrm{f}}^{(\mathrm{ac})}(\tau, \bar{\theta})+B^{(\mathrm{b})}(\tau, \bar{\theta}) \\
& \cdot\left[\Sigma^{(\mathrm{b})}(\tau, \bar{\theta}) \dot{\Sigma}^{(\mathrm{b})}(\tau, \bar{\theta})-i\left\{2 \dot{\bar{F}}^{(\mathrm{b})}(\tau, \bar{\theta}) F^{(\mathrm{b})}(\tau, \bar{\theta})\right.\right. \\
& \left.\left.+\bar{F}^{(\mathrm{b})}(\tau, \bar{\theta}) \dot{F}^{(\mathrm{b})}(\tau, \bar{\theta})\right\}\right]+\frac{1}{2} B^{(\mathrm{b})}(\tau, \bar{\theta}) B^{(\mathrm{b})}(\tau, \bar{\theta}) \\
& -i \Sigma^{(\mathrm{b})}(\tau, \bar{\theta}) \Sigma^{(\mathrm{b})}(\tau, \bar{\theta}) \dot{\bar{F}}^{(\mathrm{b})}(\tau, \bar{\theta}) \dot{F}^{(\mathrm{b})}(\tau, \bar{\theta}) \\
& -i \Sigma^{(\mathrm{b})}(\tau, \bar{\theta}) \dot{\Sigma}^{(\mathrm{b})}(\tau, \bar{\theta}) \dot{\bar{F}}^{(\mathrm{b})}(\tau, \bar{\theta}) F^{(\mathrm{b})}(\tau, \bar{\theta}) \\
& -\dot{\bar{F}}^{(\mathrm{b})}(\tau, \bar{\theta}) \bar{F}^{(\mathrm{b})}(\tau, \bar{\theta}) \dot{F}^{(\mathrm{b})}(\tau, \bar{\theta}) F^{(\mathrm{b})}(\tau, \bar{\theta}),
\end{aligned}
$$

where $\tilde{L}_{\mathrm{f}}^{(\mathrm{c})}$ and $\tilde{L}_{\mathrm{f}}^{(\mathrm{ac})}$ are the generalizations of the first-order Lagrangian $\left(L_{\mathrm{f}}\right)$ to its counterpart chiral and antichiral super Lagrangians as

$$
\begin{aligned}
\tilde{L}_{\mathrm{f}}^{(\mathrm{c})}(\tau, \theta)= & P_{x}^{(\mathrm{h}, \mathrm{c})}(\tau, \theta) \dot{X}^{(\mathrm{h}, \mathrm{c})}(\tau, \theta)+P_{t}^{(\mathrm{h}, \mathrm{c})}(\tau, \theta) \dot{T}^{(\mathrm{h}, \mathrm{c})}(\tau, \theta) \\
& -\frac{\Sigma^{(\mathrm{ab})}(\tau, \theta)}{2}\left[P_{x}^{(\mathrm{h}, \mathrm{c})}(\tau, \theta) P_{x}^{(\mathrm{h}, \mathrm{c})}(\tau, \theta)+2 m P_{t}^{(\mathrm{h}, \mathrm{c})}(\tau, \theta)\right], \\
\tilde{L}_{\mathrm{f}}^{(\mathrm{ac})}(\tau, \bar{\theta})= & P_{x}^{(\mathrm{h}, \mathrm{ac})}(\tau, \bar{\theta}) \dot{X}^{(\mathrm{h}, \mathrm{ac})}(\tau, \bar{\theta})+P_{t}^{(\mathrm{h}, \mathrm{ac})}(\tau, \bar{\theta}) \dot{T}^{(\mathrm{h}, \mathrm{acc})} \\
\cdot(\tau, \bar{\theta})- & \frac{\Sigma^{(\mathrm{b})}(\tau, \bar{\theta})}{2}\left[P_{x}^{(\mathrm{h}, \mathrm{acc})}(\tau, \bar{\theta}) P_{x}^{(\mathrm{h}, \mathrm{ac})}(\tau, \bar{\theta})+2 m P_{t}^{(\mathrm{h}, \mathrm{ac})}(\tau, \bar{\theta})\right],
\end{aligned}
$$

where the superscripts (c) and (ac) denote the chiral and antichiral generalizations and the rest of the supervariables with superscripts (b) and (ab) have already been explained earlier in Section 5. The supervariables with superscripts $(h, c)$ and ( $\mathrm{h}, \mathrm{ac}$ ) are the chiral and antichiral limits of the super phase variables $\left(X^{(\mathrm{h})}, P_{x}^{(\mathrm{h})}, T^{(\mathrm{h})}\right.$, and $\left.P_{t}^{(\mathrm{h})}\right)$ that have been obtained after the application of HC. Thus, these are the counterparts of the ordinary phase variables $\left(x, p_{x}, t\right.$, and $\left.p_{t}\right)$ and they have been explained in Section 3. In the above Equation (65), the super phase variables with superscript $(h, c)$ and $(h, a c)$ can be expressed in terms of the generic supervariable as follows:

$$
\begin{aligned}
S(\tau) \longrightarrow S^{(\mathrm{h}, \mathrm{c})}(\tau, \theta) & =S(\tau)+\theta[\bar{C} \dot{S}(\tau)] \\
S(\tau) \longrightarrow S^{(\mathrm{h}, \mathrm{ac})}(\tau, \bar{\theta}) & =S(\tau)+\bar{\theta}[C \dot{S}(\tau)]
\end{aligned}
$$

where the (anti)chiral supervariables on the l.h.s. stand for the super phase variables $\left(X, P_{x}, T\right.$, and $\left.P_{t}\right)$ with the proper chiral and antichiral superspace coordinates $(\tau, \theta)$ and $(\tau, \bar{\theta})$ as their arguments. The set of supervariables $\left(X, P_{x}, T\right.$, and $\left.P_{t}\right)$ is the generalization of the ordinary phase variables $\left(x, p_{x}, t\right.$, and $\left.p_{t}\right)$ to their (anti)chiral counterparts onto the $(1,1)$-dimensional (anti)chiral super submanifolds of the general $(1,2)$-dimensional supermanifold. It is straightforward to check that the following is true, namely,

$$
\begin{gathered}
\frac{\partial}{\partial \theta} \tilde{L}_{\mathrm{f}}^{(\mathrm{c})}(\tau, \theta)=\frac{d}{d \tau}\left[\bar{C} L_{\mathrm{f}}\right] \Leftrightarrow s_{a b} L_{\mathrm{f}}=\frac{d}{d \tau}\left[\bar{C} L_{\mathrm{f}}\right], \\
\frac{\partial}{\partial \bar{\theta}} \tilde{L}_{\mathrm{f}}^{\text {(ac) }}(\tau, \bar{\theta})=\frac{d}{d \tau}\left[C L_{\mathrm{f}}\right] \Leftrightarrow s_{b} L_{\mathrm{f}}=\frac{d}{d \tau}\left[C L_{\mathrm{f}}\right] .
\end{gathered}
$$

In other words, we have captured the (anti-)BRST invariance of the first-order Lagrangian $\left(L_{\mathrm{f}}\right)$ in view of the mappings $s_{b} \leftrightarrow \partial_{\bar{\theta}}$ and $s_{a b} \leftrightarrow \partial_{\theta}$. Since in the ordinary space the (anti-)BRST symmetry transformations acting on $L_{\mathrm{f}}$ produce the total derivatives (cf. Equation (67)), the action integral $S=\int_{-\infty}^{+\infty} d \tau L_{\mathrm{f}}$ remains invariant under the transformations $s_{(a) b}$.

At this stage, we focus on the (anti-)BRST invariance of the coupled Lagrangians $L_{B}$ and $L_{\bar{B}}$ (cf. Equations (37) and (38)). We can express these invariances within the ambit of ACSA (in view of the mappings $s_{b} \leftrightarrow \partial_{\bar{\theta}}$ and $s_{a b} \leftrightarrow \partial_{\theta}$ ), namely,

$$
\begin{aligned}
& \frac{\partial}{\partial \theta} \tilde{L}_{\bar{B}}^{(\mathrm{c})}(\tau, \theta)=\frac{d}{d \tau}\left[\bar{C} L_{\mathrm{f}}-e \dot{e} \bar{B} \bar{C}-e^{2} \overline{\bar{B}} \dot{\bar{C}}+\bar{B}^{2} \bar{C}-i \bar{B} \dot{\bar{C}} \bar{C} C\right]=s_{a b} L_{\bar{B}} \\
& \frac{\partial}{\partial \bar{\theta}} \tilde{L}_{B}^{(\text {ac) }}(\tau, \bar{\theta})=\frac{d}{d \tau}\left[C L_{\mathrm{f}}+e \dot{e} B C+e^{2} B \dot{C}+B^{2} C-i B \bar{C} \dot{C} C\right]=s_{b} L_{B}
\end{aligned}
$$

where the super Lagrangians $\tilde{L}_{\bar{B}}^{(\mathrm{c})}(\tau, \theta)$ and $\tilde{L}_{B}^{(\text {ac })}(\tau, \bar{\theta})$ have been already quoted in Equations (63) and (64). It is interesting to note that the r.h.s. of (68) and (69) are the same as we have found in the ordinary space (cf. Equations (37) and (38)). To prove the equivalence of the Lagrangians $L_{B}$ and $L_{\bar{B}}$ w.r.t. the (anti-)BRST symmetry transformations (cf. Equations (32) and (33)) within the purview of ACSA, we generalize the ordinary Lagrangians $L_{B}$ and $L_{\bar{B}}$ as follows: 


$$
\begin{aligned}
& L_{B} \longrightarrow \tilde{L}_{B}^{(\mathrm{c})}(\tau, \theta)=\tilde{L}_{\mathrm{f}}^{(\mathrm{c})}(\tau, \theta)+B^{(\mathrm{ab})}(\tau, \theta) {\left[\Sigma^{(\mathrm{ab})}(\tau, \theta) \dot{\Sigma}^{(\mathrm{ab})}(\tau, \theta)-i\left\{2 \dot{\bar{F}}^{(\mathrm{ab})}(\tau, \theta) F^{(\mathrm{ab})}(\tau, \theta)\right.\right.} \\
&\left.\left.+\bar{F}^{(\mathrm{ab})}(\tau, \theta) \dot{F}^{(\mathrm{ab})}(\tau, \theta)\right\}\right]+\frac{1}{2} B^{(\mathrm{ab})}(\tau, \theta) B^{(\mathrm{ab})}(\tau, \theta) \\
&-i \Sigma^{(\mathrm{ab})}(\tau, \theta) \Sigma^{(\mathrm{ab})}(\tau, \theta) \dot{\bar{F}}^{(\mathrm{ab})}(\tau, \theta) \dot{F}^{(\mathrm{ab})}(\tau, \theta) \\
&-i \Sigma^{(\mathrm{ab})}(\tau, \theta) \dot{\Sigma}^{(\mathrm{ab})}(\tau, \theta) \dot{\bar{F}}^{(\mathrm{ab})}(\tau, \theta) F^{(\mathrm{ab})}(\tau, \theta) \\
&-\dot{\bar{F}}^{(\mathrm{ab})}(\tau, \theta) \bar{F}^{(\mathrm{ab})}(\tau, \theta) \dot{F}^{(\mathrm{ab})}(\tau, \theta) F^{(\mathrm{ab})}(\tau, \theta) \\
& L_{\bar{B}} \longrightarrow \tilde{L}_{\bar{B}}^{(\mathrm{ac})}(\tau, \bar{\theta})=\tilde{L}_{\mathrm{f}}^{(\mathrm{ac})}(\tau, \bar{\theta})-B^{(\mathrm{b})}(\tau, \bar{\theta}) \\
& \cdot\left[\Sigma^{(\mathrm{b})}(\tau, \bar{\theta}) \dot{\Sigma}^{(\mathrm{b})}(\tau, \bar{\theta})-i\left\{2 \bar{F}^{(\mathrm{b})}(\tau, \bar{\theta}) \dot{F}^{(\mathrm{b})}(\tau, \bar{\theta})+\dot{\bar{F}}^{(\mathrm{b})}\right.\right. \\
&\left.\left.\cdot(\tau, \bar{\theta}) F^{(\mathrm{b})}(\tau, \bar{\theta})\right\}\right]+\frac{1}{2} B^{(\mathrm{b})}(\tau, \bar{\theta}) B^{(\mathrm{b})}(\tau, \bar{\theta}) \\
&-i \Sigma^{(\mathrm{b})}(\tau, \bar{\theta}) \Sigma^{(\mathrm{b})}(\tau, \bar{\theta}) \dot{\bar{F}}^{(\mathrm{b})}(\tau, \bar{\theta}) \dot{F}^{(\mathrm{b})}(\tau, \bar{\theta}) \\
&-i \Sigma^{(\mathrm{b})}(\tau, \bar{\theta}) \dot{\Sigma}^{(\mathrm{b})}(\tau, \bar{\theta}) \bar{F}^{(\mathrm{b})}(\tau, \bar{\theta}) \dot{F}^{(\mathrm{b})}(\tau, \bar{\theta}) \\
&-\dot{\bar{F}}^{(\mathrm{b})}(\tau, \bar{\theta}) \bar{F}^{(\mathrm{b})}(\tau, \bar{\theta}) \dot{F}^{(\mathrm{b})}(\tau, \bar{\theta}) F^{(\mathrm{b})}(\tau, \bar{\theta}) \\
&
\end{aligned}
$$

where all the notations and symbols have already been explained earlier. To find out the result of the operations of $s_{b}$ on $L_{\bar{B}}$ and $s_{a b}$ on $L_{B}$, we observe the following (in view of the mappings $s_{b} \leftrightarrow \partial_{\bar{\theta}}$ and $s_{a b} \leftrightarrow \partial_{\theta}$ ), namely,

$$
\begin{aligned}
& \frac{\partial}{\partial \theta} \tilde{L}_{B}^{(\mathrm{c})}(\tau, \theta)=\frac{d}{d \tau}\left[\bar{C} L_{\mathrm{f}}+E \dot{E}(i \dot{\bar{C}} \bar{C} C+B \bar{C})\right. \\
& \left.+E^{2}(i \dot{\bar{C}} \bar{C} \dot{C}+B \dot{\bar{C}})+B^{2} \bar{C}+i(2 B-\bar{B}) \dot{\bar{C}} \bar{C} C\right] \\
& +[B+\bar{B}+i(\bar{C} \dot{C}-\dot{\bar{C}} C)](2 i \dot{\bar{C}} \bar{C} \dot{C}-E \dot{\bar{E}} \dot{\bar{C}}-2 B \dot{\bar{C}}+i \ddot{\bar{C}} \bar{C} C) \\
& \quad-\frac{d}{d \tau}[B+\bar{B}+i(\bar{C} \dot{C}-\dot{\bar{C}} C)]\left(B \bar{C}+E^{2} \dot{\bar{C}}\right) \equiv s_{a b} L_{B},
\end{aligned}
$$

$$
\begin{aligned}
& \frac{\partial}{\partial \bar{\theta}} \tilde{L}_{\bar{B}}^{(\mathrm{ac})}(\tau, \bar{\theta})=\frac{d}{d \tau}\left[C L_{\mathrm{f}}-E \dot{E}(i \bar{C} \dot{C} C+\bar{B} C)\right. \\
& \left.\quad-E^{2}(i \dot{\bar{C}} \dot{C} C+\bar{B} \dot{C})+\bar{B}^{2} C+i(2 \bar{B}-B) \bar{C} \dot{C} C\right] \\
& \quad+[B+\bar{B}+i(\bar{C} \dot{C}-\dot{\bar{C}} C)][i \bar{C} \ddot{C} C+2 i \dot{\bar{C}} \dot{C} C-2 \bar{B} \dot{C}+E \dot{E} \dot{C}] \\
& \quad+\frac{d}{d \tau}[i(\bar{C} \dot{C}-\dot{\bar{C}} C)+B+\bar{B}]\left(E^{2} \dot{C}-\bar{B} C\right) \equiv s_{b} L_{\bar{B}}
\end{aligned}
$$

within the framework of ACSA. It is self-evident, from the r.h.s. of (72) and (73), that we have the BRST invariance of $L_{\bar{B}}$ and anti-BRST invariance of $L_{B}$ if and only if our whole theory is considered on the submanifold of the Hilbert space of quantum variables where the CF-type restriction $B+\bar{B}+i(\bar{C} \dot{C}-\dot{\bar{C}} C)=0$ is satisfied.
We end this section with the following crucial remarks. First of all, we have captured the BRST and anti-BRST invariances of $L_{B}$ and $L_{\bar{B}}$, respectively, in the terminology of ACSA on the (anti)chiral super submanifolds (cf. Equations (68) and (69)). Second, we have also demonstrated the antiBRST invariance of $L_{B}$ and BRST invariance of $L_{\bar{B}}$ in the superspace formalism (cf. Equations (72) and (73)) where the theoretical techniques of ACSA have played very important roles. Third, we have also expressed the (anti-)BRST invariance of the first-order Lagrangian $L_{\mathrm{f}}$ in Equation (67). Finally, we have proven the equivalence of $L_{B}$ and $L_{\bar{B}}$ within the framework of ACSA in Equations (68), (69), (72), and (73).

\section{Nilpotency and Absolute Anticommutativity Properties of the (Anti-)BRST Charges: ACSA}

Our present section is divided into two sections. In Section 7.1, we discuss the off-shell nilpotency and absolute anticommutativity of the conserved (anti-)BRST charges in the ordinary space. Section 7.2 deals with the above properties within the realm of ACSA to BRST formalism. In other words, we capture the off-shell nilpotency and absolute anticommutativity of the conserved fermionic (anti-)BRST charges in the superspace by taking the theoretical inputs from ACSA.

7.1. Nilpotency and Anticommutativity: Ordinary Space. The perfect symmetry invariances of $L_{\bar{B}}$ under the anti-BRST symmetry transformations (cf. Equation (38)) and $L_{B}$ under the BRST symmetry transformations (cf. Equation (37)) allow us to compute the Noether conserved charges by using the standard techniques of the Noether theorem (applied to the action integrals corresponding to the Lagrangians $L_{\bar{B}}$ and $L_{B}$ ) as

$$
\begin{aligned}
Q_{\bar{B}} & =\frac{\bar{C} E}{2}\left(p_{x}^{2}+2 m p_{t}\right)+\bar{B}^{2} \bar{C}-i \overline{\bar{B}} \dot{\bar{C}} \bar{C} C-\bar{B} E \dot{E} \bar{C}-\bar{B} E^{2} \dot{\bar{C}}, \\
Q_{B} & =\frac{C E}{2}\left(p_{x}^{2}+2 m p_{t}\right)+B E^{2} \dot{C}-i B \bar{C} \dot{C} C+B E \dot{E} C+B^{2} C,
\end{aligned}
$$

where conserved $\left(\dot{Q}_{\bar{B}}=0, \dot{Q}_{B}=0\right)$ (anti-)BRST charges are denoted by $Q_{(\bar{B}) B}$. The conservation law $\left(\dot{Q}_{\bar{B}}=0, \dot{Q}_{B}=0\right)$ can be proven by using the EL-EOMs derived from the coupled Lagrangians $L_{\bar{B}}$ and $L_{B}$. For readers' convenience, we prove the conservation $\left(\dot{Q}_{B}=0\right)$ of the BRST charge by using the EL-EOMs derived from $L_{B}$ in Appendix C.

First of all, we concentrate on the proof of the off-shell nilpotency properties of the (anti-)BRST charges $Q_{(\bar{B}) B}$. In this context, we note that the following EL-EOMs w.r.t. the variable $E$ from $L_{\bar{B}}$ and $L_{B}$, respectively, yield the following:

$$
\begin{aligned}
& \dot{\bar{B}} E-i E \dot{\bar{C}} \dot{C}+i E \bar{C} \ddot{C}-\frac{1}{2}\left(p_{x}^{2}+2 m p_{t}\right)=0, \\
& \dot{B} E+i E \dot{\bar{C}} \dot{C}-i E \ddot{\bar{C}} C+\frac{1}{2}\left(p_{x}^{2}+2 m p_{t}\right)=0
\end{aligned}
$$



follows:

The above equations can be used to recast $Q_{\bar{B}}$ and $Q_{B}$ as

$$
\begin{aligned}
& Q_{\bar{B}}^{(1)}=E^{2}(\dot{\bar{B}} \bar{C}-\bar{B} \dot{\bar{C}}+i \dot{\bar{C}} \bar{C} \dot{C})-i \bar{B} \dot{\bar{C}} \bar{C} C-\bar{B} E \dot{E} \bar{C}+\bar{B}^{2} \bar{C}, \\
& Q_{B}^{(1)}=E^{2}(B \dot{C}-\dot{B} C-i \dot{\bar{C}} \dot{C} C)-i B \bar{C} \dot{C} C+B E \dot{E} C+B^{2} C .
\end{aligned}
$$

Using the following EL-EOMs w.r.t. the variables $C$ and $\bar{B}$, respectively, from $L_{\bar{B}}$, namely,

$$
\begin{gathered}
i \overline{\bar{B}} \dot{\bar{C}}+2 i \dot{\bar{B}} \bar{C}-3 i E \dot{E} \dot{\bar{C}}-i E^{2} \ddot{\bar{C}}-i \dot{E}^{2} \bar{C}-i E \ddot{E} \bar{C}+\ddot{\bar{C}} \bar{C} C+2 \dot{\bar{C}} \bar{C} \dot{C}=0, \\
\bar{B}=E \dot{E}-i(2 \bar{C} \dot{C}+\dot{\bar{C}} C),
\end{gathered}
$$

we obtain the following exact and interesting expression for the anti-BRST charge:

$$
\begin{aligned}
& Q_{\bar{B}}^{(1)} \longrightarrow Q_{\bar{B}}^{(2)}=E^{2}(\dot{\bar{B}} \bar{C}-\bar{B} \dot{\bar{C}}+i \dot{\bar{C}} \bar{C} \dot{C})+i E^{2} \ddot{\bar{C}} \bar{C} C \\
& \quad+2 i E \dot{E} \dot{\bar{C}} \bar{C} C \equiv s_{a b}\left[i E^{2}(\bar{C} \dot{C}-\dot{\bar{C}} C)\right] .
\end{aligned}
$$

At this juncture, we apply the basic principle behind the relationship between the continuous symmetry transformations (e.g., $s_{a b}$ ) and its generator $\left[Q_{\bar{B}}^{(2)}\right]$ which implies that

$$
s_{a b} Q_{\bar{B}}^{(2)}=-i\left\{Q_{\bar{B}}^{(2)}, Q_{\bar{B}}^{(2)}\right\}=0 \Rightarrow\left[Q_{\bar{B}}^{(2)}\right]^{2}=0 \Leftrightarrow s_{a b}^{2}=0 .
$$

Thus, we observe that the off-shell nilpotency $\left(\left[Q_{\bar{B}}^{(2)}\right]^{2}\right.$ $=0)$ of the anti-BRST charge $Q_{\bar{B}}^{(2)}$ and the anti-BRST symmetry transformations $\left(s_{a b}\right)$ are interrelated. Thus, we have proven the off-shell nilpotency of the anti-BRST charge $Q_{\bar{B}}^{(2)}$. In an exactly similar fashion, we exploit the following EL-EOMs w.r.t. the variables $\bar{C}$ and $B$ from the Lagrangian $L_{B}$ :

$$
\begin{gathered}
i B \dot{C}+2 i \dot{B} C+3 i \dot{E} \dot{E} \dot{C}+i E^{2} \ddot{C}+i \dot{E}^{2} C+i E \ddot{E} C+\bar{C} \ddot{C} C+2 \dot{\bar{C}} \dot{C} C=0, \\
B=-E \dot{E}+i(2 \dot{\bar{C}} C+\bar{C} \dot{C}),
\end{gathered}
$$

to recast the BRST charge $Q_{B}^{(1)}$ into another interesting form (i.e., $Q_{B}^{(2)}$ ) as

$$
\begin{aligned}
& Q_{B}^{(1)} \longrightarrow Q_{B}^{(2)}=E^{2}(B \dot{C}-i \dot{\bar{C}} \dot{C} C-\dot{B} C)-2 i E \dot{E} \bar{C} \dot{C} C-i E^{2} \bar{C} \ddot{C} C \\
& \quad \equiv s_{b}\left[i E^{2}(\dot{\bar{C}} C-\bar{C} \dot{C})\right],
\end{aligned}
$$

which turns out to be an exact quantity w.r.t. $s_{b}$. Thus, we find that we have the following:

$$
s_{b} Q_{B}^{(2)}=-i\left\{Q_{B}^{(2)}, Q_{B}^{(2)}\right\}=0 \Rightarrow\left[Q_{B}^{(2)}\right]^{2}=0 \Leftrightarrow s_{b}^{2}=0 .
$$

In other words, we have proven the off-shell nilpotency $\left(\left[Q_{B}^{(2)}\right]^{2}=0\right)$ of the BRST charge $Q_{B}^{(2)}$. Once again, we find that the off-shell nilpotency $\left(s_{b}^{2}=0\right)$ of the BRST symmetry transformations and the off-shell nilpotency $\left(\left[Q_{B}^{(2)}\right]^{2}=0\right)$ are intertwined in an intimate manner.

We now focus on the proof of the absolute anticommutativity property of the BRST charge with the anti-BRST charge and vice versa. First of all, let us focus on the BRST charge $Q_{B}^{(2)}$ (cf. Equation (81)). Using the CF-type restriction $B+\bar{B}+i(\bar{C} \dot{C}-$ $\dot{\bar{C}} C)=0$, we can easily check the following transformation:

$$
Q_{B}^{(2)} \longrightarrow Q_{B}^{(3)}=E^{2}(\dot{\bar{B}} C-2 i \dot{\bar{C}} \dot{C} C-\bar{B} \dot{C})-2 i E \dot{E} \bar{C} \dot{C} C \equiv s_{a b}\left[i E^{2} \dot{C} C\right]
$$

In other words, we have been able to express the above BRST charge as an exact form w.r.t. the anti-BRST symmetry transformations $\left(s_{a b}\right)$. This is an interesting observation because using the relationship between the continuous symmetry transformations and their generators, we can obtain the following from (83), namely,

$$
s_{a b} Q_{B}^{(3)}=-i\left\{Q_{B}^{(3)}, Q_{\bar{B}}^{(3)}\right\}=0 \Leftrightarrow s_{a b}^{2}=0 .
$$

Thus, we have been able to demonstrate that the absolute anticommutativity of the BRST charge with the anti-BRST charge is connected with the off-shell nilpotency $\left(s_{a b}^{2}=0\right)$ of the anti-BRST symmetry transformation $\left(s_{a b}\right)$. In an exactly similar fashion, we can have a different form of the anti-BRST charge $Q_{\bar{B}}^{(2)}$ (cf. Equation (78)) by using the CF-type restriction $B+\bar{B}+i(\bar{C} \dot{C}-\dot{\bar{C}} C)=0$. In other words, we have the following interesting transformation:

$$
Q_{\bar{B}}^{(3)} \longrightarrow Q_{\bar{B}}^{(3)}=E^{2}(B \dot{\bar{C}}+2 i \dot{\bar{C}} \bar{C} \dot{C}-\dot{B} \bar{C})+2 i E \dot{E} \dot{\bar{C}} \bar{C} C \equiv s_{b}\left[i E^{2} \dot{\bar{C}} \bar{C}\right] .
$$

It is straightforward to note that we have the following relationship:

$$
s_{b} Q_{\bar{B}}^{(3)}=-i\left\{Q_{\bar{B}}^{(3)}, Q_{B}^{(3)}\right\}=0 \Leftrightarrow s_{b}^{2}=0 .
$$

In other words, we point out that the absolute anticommutativity of the anti-BRST charge with the BRST charge is intimately connected with the off-shell nilpotency $\left(s_{b}^{2}=0\right)$ of the BRST symmetry transformations $\left(s_{b}\right)$. This completes our discussions on the off-shell nilpotency and absolute anticommutativity of the conserved (anti-)BRST charges in the ordinary 
space. In a subtle manner, the observations in (83) and (85) prove the validity of the CF-type restriction $B+\bar{B}+i(\bar{C} \dot{C}-\dot{\bar{C}}$ $C)=0$ on our theory.

7.2. Nilpotency and Anticommutativity: ACSA. The key observations of Section 7.1 can be translated into the superspace by using the basic terminology of ACSA. Keeping in our mind the mappings $\partial_{\bar{\theta}} \leftrightarrow s_{b}$ and $\partial_{\theta} \leftrightarrow s_{a b}$, we note that the (anti-)BRST charges $Q_{(\bar{B}) B}$ (cf. Equations (78) and (81)) can be expressed as

$$
\begin{aligned}
Q_{\bar{B}}= & \frac{\partial}{\partial \theta}\left[i E ^ { ( \mathrm { ab } ) } ( \tau , \theta ) E ^ { ( \mathrm { ab } ) } ( \tau , \theta ) \left\{\bar{F}^{(\mathrm{ab})}(\tau, \theta) \dot{F}^{(\mathrm{ab})}(\tau, \theta)\right.\right. \\
& \left.\left.-\dot{\bar{F}}^{(\mathrm{ab})}(\tau, \theta) F^{(\mathrm{ab})}(\tau, \theta)\right\}\right]=\int d \theta\left[i E^{(\mathrm{ab})}(\tau, \theta) E^{(\mathrm{ab})}\right. \\
& \left.\cdot(\tau, \theta)\left\{\bar{F}^{(\mathrm{ab})}(\tau, \theta) \dot{F}^{(\mathrm{ab})}(\tau, \theta)-\dot{\bar{F}}^{(\mathrm{ab})}(\tau, \theta) F^{(\mathrm{ab})}(\tau, \theta)\right\}\right],
\end{aligned}
$$

$$
\begin{aligned}
Q_{B}= & \frac{\partial}{\partial \bar{\theta}}\left[i E ^ { ( \mathrm { b } ) } ( \tau , \overline { \theta } ) E ^ { ( \mathrm { b } ) } ( \tau , \overline { \theta } ) \left\{\dot{\bar{F}}^{(\mathrm{b})}(\tau, \bar{\theta}) F^{(\mathrm{b})}(\tau, \bar{\theta})\right.\right. \\
& \left.\left.-\bar{F}^{(\mathrm{b})}(\tau, \bar{\theta}) \dot{F}^{(\mathrm{b})}(\tau, \bar{\theta})\right\}\right]=\int d \bar{\theta}\left[i E^{(\mathrm{b})}(\tau, \bar{\theta}) E^{(\mathrm{b})}\right. \\
& \left.\cdot(\tau, \bar{\theta})\left\{\dot{\bar{F}}^{(\mathrm{b})}(\tau, \bar{\theta}) F^{(\mathrm{b})}(\tau, \bar{\theta})-\bar{F}^{(\mathrm{b})}(\tau, \bar{\theta}) \dot{F}^{(\mathrm{b})}(\tau, \bar{\theta})\right\}\right] .
\end{aligned}
$$

It is straightforward to observe that we have the following:

$$
\begin{gathered}
\frac{\partial}{\partial \theta} Q_{\bar{B}}=0 \Leftrightarrow s_{a b} Q_{\bar{B}}=0 \Leftrightarrow Q_{\bar{B}}^{2}=0 \Leftrightarrow \partial_{\theta}^{2}=0, \\
\frac{\partial}{\partial \bar{\theta}} Q_{B}=0 \Leftrightarrow s_{b} Q_{B}=0 \Leftrightarrow Q_{B}^{2}=0 \Leftrightarrow \partial_{\bar{\theta}}^{2}=0 .
\end{gathered}
$$

Thus, the off-shell nilpotency of the (anti-)BRST charges is connected with the nilpotency $\left(\partial_{\theta}^{2}=0, \partial_{\bar{\theta}}^{2}=0\right)$ of the translational generators $\left(\partial_{\theta}, \partial_{\bar{\theta}}\right)$ along the Grassmannian directions of the chiral and antichiral $(1,1)$-dimensional super submanifolds. This observation is consistent with our discussion of the nilpotency property in the ordinary space if we remember the mappings $s_{b} \leftrightarrow \partial_{\bar{\theta}}$ and $s_{a b} \leftrightarrow \partial_{\theta}$ [14-16].

As far as the absolute anticommutativity property is concerned, we note that the expressions of the (anti-)BRST charges in (83) and (85) can be translated into the superspace where we can exploit the theoretical tools of ACSA. To accomplish this goal, we keep in our knowledge the mappings $s_{b} \leftrightarrow \partial_{\bar{\theta}}$ and $s_{a b} \leftrightarrow \partial_{\theta}$ to recast the expressions (83) and (85) as

$$
\begin{aligned}
Q_{\bar{B}}^{(3)} & =\frac{\partial}{\partial \bar{\theta}}\left[i E^{(\mathrm{b})}(\tau, \bar{\theta}) E^{(\mathrm{b})}(\tau, \bar{\theta}) \dot{\bar{F}}^{(\mathrm{b})}(\tau, \bar{\theta}) \bar{F}^{(\mathrm{b})}(\tau, \bar{\theta})\right] \\
& \equiv \int d \bar{\theta}\left[i E^{(\mathrm{b})}(\tau, \bar{\theta}) E^{(\mathrm{b})}(\tau, \bar{\theta}) \dot{\bar{F}}^{(\mathrm{b})}(\tau, \bar{\theta}) \bar{F}^{(\mathrm{b})}(\tau, \bar{\theta})\right], \\
Q_{B}^{(3)} & =\frac{\partial}{\partial \theta}\left[-i E^{(\mathrm{ab})}(\tau, \theta) E^{(\mathrm{ab})}(\tau, \theta) \dot{F}^{(\mathrm{ab})}(\tau, \theta) F^{(\mathrm{ab})}(\tau, \theta)\right] \\
& \equiv \int d \theta\left[-i E^{(\mathrm{ab})}(\tau, \theta) E^{(\mathrm{ab})}(\tau, \theta) \dot{F}^{(\mathrm{ab})}(\tau, \theta) F^{(\mathrm{ab})}(\tau, \theta)\right] .
\end{aligned}
$$

It is now straightforward to check that the following are true, namely,

$$
\begin{aligned}
& \partial_{\bar{\theta}} Q_{\bar{B}}^{(3)}=0 \Leftrightarrow s_{b} Q_{\bar{B}}^{(3)}=0 \Leftrightarrow\left\{Q_{\bar{B}}^{(3)}, Q_{B}^{(3)}\right\}=0 \Leftrightarrow \partial_{\bar{\theta}}^{2}=0, \\
& \partial_{\theta} Q_{B}^{(3)}=0 \Leftrightarrow s_{a b} Q_{B}^{(3)}=0 \Leftrightarrow\left\{Q_{B}^{(3)}, Q_{\bar{B}}^{(3)}\right\}=0 \Leftrightarrow \partial_{\theta}^{2}=0,
\end{aligned}
$$

which establishes the fact that the ACSA to BRST formalism distinguishes between the two types of absolute anticommutativity properties. In other words, we observe that the absolute anticommutativity of the BRST charge with the antiBRST charge is connected with the nilpotency $\left(\partial_{\theta}^{2}=0\right)$ of the translational generator $\left(\partial_{\theta}\right)$ along the Grassmannian direction of the $(1,1)$-dimensional chiral super submanifold. On the contrary, the absolute anticommutativity of the antiBRST charge with the BRST charge is connected with the nilpotency $\left(\partial_{\bar{\theta}}^{2}=0\right)$ of the translational generator $\left(\partial_{\bar{\theta}}\right)$ along the Grassmannian direction of the $(1,1)$-dimensional antichiral super submanifold.

\section{Conclusions}

In our present endeavor, we have purposely taken a reparameterization invariant NR and NSUSY system so that we could discuss theoretical aspects that are different from our earlier works on the NSUSY relativistic scalar and SUSY relativistic spinning particles $[23,24]$. We have demonstrated, however, in our present investigation that (i) the CF-type restriction and (ii) the sum of gauge-fixing and FaddeevPopov ghost terms are the same for our present NR and NSUSY system as have been shown by us for the relativistic particles (in our earlier works $[23,24]$ ). The above observations are interesting results of our present investigation which establish the universality of the (anti-)BRST invariant CF-type restriction for the $1 \mathrm{D}$ diffeomorphism invariant (i.e., reparameterization) theories.

The CF-type restriction(s) are the hallmark of a quantum theory that is BRST-quantized. In fact, for a D-dimensional diffeomorphism invariant theory, it has been shown [22, 33] that the universal CF-type restriction for a BRST-quantized theory is $B_{\mu}+\bar{B}_{\mu}+i\left(\bar{C}^{\rho} \partial_{\rho} C_{\mu}+C^{\rho} \partial_{\rho} \bar{C}_{\mu}\right)=0$, where $\mu, \rho=0,1,2, \cdots, D-1, B_{\mu}$ and $\bar{B}_{\mu}$ are the NakanishiLautrup auxiliary fields, and the (anti)ghost fields $\left(\bar{C}_{\mu}\right) C_{\mu}$ correspond to the $D$-dimensional diffeomorphism parameter 
$\varepsilon_{\mu}(x)$ in the infinitesimal transformation $x_{\mu} \longrightarrow x_{\mu}{ }^{\prime}=x_{\mu}-$ $\varepsilon_{\mu}(x)$. The universality of the above CF-type restriction implies that, for our 1D diffeomorphism (i.e., reparameterization) invariant theory, the CF-type restriction is $B+\bar{B}+i$ $(\bar{C} \dot{C}-\dot{\bar{C}} C)=0$. This is what we have obtained from various theoretical tricks in our present endeavor. The existence of the CF-type restriction is very fundamental to a BRSTquantized theory as it is connected with the geometrical objects called gerbes [7, 8]. Physically, the existence of the CF-type restriction leads to the independent nature of the BRST and anti-BRST symmetries (and corresponding conserved charges) at the quantum level (that are connected with a given classical local symmetry).

Our present work (and earlier works $[23,24]$ ) can be generalized to the cases of (super)string and gravitational theories which are also diffeomorphism invariant. In fact, in our earlier work on a bosonic string theory [34], we have shown the existence of the CF-type restriction in the context of its BRST quantization and it has turned out to be the $2 \mathrm{D}$ version of the universal CF-type restriction for the $D$-dimensional diffeomorphism invariant theory. It is gratifying to pinpoint the fact that we have derived the CF-type restrictions $B^{a}+\bar{B}^{a}+i\left(\bar{C}^{m}\right.$ $\partial_{m} C^{a}+C^{m} \partial_{m} \bar{C}^{a}$ ) $=0$ (with $a, m=0,1$ ) for a model of bosonic string theory [34]. This has happened because the bosonic string theory has the $2 \mathrm{D}$ diffeomorphism invariance on the 2D worldsheet. We have applied the beautiful blend of MBTSA and ACSA to derive all the (anti-)BRST symmetries as well as the $2 \mathrm{D}$ version of the CF-type restriction in the case of a bosonic string theory of our interest [35]. In our present investigation, we have utilized only one and/or two Grassmannian variables because there are only two nilpotent symmetries in the theory. If a theory is endowed with the nilpotent (anti-)BRST as well as (anti-)co-BRST symmetries, then we have to invoke four numbers of the Grassmannian variables. We are currently exploring such kinds of possibilities.

\section{Appendix}

\section{A. The CF-Type Restriction from $L_{B} \equiv L_{\bar{B}}$}

In this appendix, we provide the step-by-step derivation of the CF-type restriction by requiring the equivalence of the coupled Lagrangians $L_{B}$ and $L_{\bar{B}}$ (cf. Equation (35)). A close look at them demonstrates that if we demand $L_{B} \equiv L_{\bar{B}}$, the terms that are common would cancel out. For instance, we have cancelations of terms $L_{\mathrm{f}},-i E^{2} \dot{\bar{C}} \dot{C}$, and $-\dot{\bar{C}} \bar{C} \dot{C} C$ that are present in both $L_{B}$ and $L_{\bar{B}}$. Thus, we are left with the following equality:

$$
\begin{aligned}
\frac{B^{2}}{2}+B[E \dot{E}-i(2 \dot{\bar{C}} C+\bar{C} \dot{C})]-i E \dot{E} \dot{\bar{C}} C \equiv \frac{\bar{B}^{2}}{2} \\
+\bar{B}[E \dot{E}-i(2 \bar{C} \dot{C}+\dot{\bar{C}} C)]-i E \dot{E} \bar{C} \dot{C}
\end{aligned}
$$

From the above equation, it is evident that we have

$$
E \dot{E}[B+\bar{B}+i(\bar{C} \dot{C}-\dot{\bar{C}} C)]
$$

on the l.h.s. when we bring all the terms from the r.h.s. to the l.h.s. At this stage, excluding (A.2), the leftover terms on the l.h.s. and the r.h.s. are

$$
\frac{B^{2}}{2}-\frac{\bar{B}^{2}}{2}-2 i B \dot{\bar{C}} C-i B \bar{C} \dot{C}-2 i \bar{B} \bar{C} \dot{C}-i \bar{B} \dot{\bar{C}} C=0
$$

The above equation can be expressed as

$$
\frac{B^{2}}{2}-\frac{\bar{B}^{2}}{2}-i(B+\bar{B}) \dot{\bar{C}} C-i(B+\bar{B}) \bar{C} \dot{C}-i B \dot{\bar{C}} C-i \bar{B} \bar{C} \dot{C}=0
$$

The rearrangements of the terms produce the following:

$$
\begin{aligned}
\frac{B^{2}}{2}- & \frac{\bar{B}^{2}}{2}-i[B+\bar{B}+i(\bar{C} \dot{C}-\dot{\bar{C}} C)] \dot{\bar{C}} C \\
& -i[B+\bar{B}+i(\bar{C} \dot{C}-\dot{\bar{C}} C)] \bar{C} \dot{C}-i B \dot{\bar{C}} C-i \bar{B} \bar{C} \dot{C}=0 .
\end{aligned}
$$

Taking into account (A.2), we have the following:

$$
\begin{gathered}
{[E \dot{E}-i \dot{\bar{C}} C-i \bar{C} \dot{C}][B+\bar{B}+i(\bar{C} \dot{C}-\dot{\bar{C}} C)]} \\
+\frac{B^{2}}{2}-\frac{\bar{B}^{2}}{2}-i B \dot{\bar{C}} C-i \bar{B} \bar{C} \dot{C}=0 .
\end{gathered}
$$

Substituting for $-i \dot{\bar{B}} C=-(i / 2) B \dot{\bar{C}} C-(i / 2) B \dot{\bar{C}} C$ and $-i$ $\bar{B} \bar{C} \dot{C}=-(i / 2) \bar{B} \bar{C} \dot{C}-(i / 2) \bar{B} \bar{C} \dot{C}$ and rearranging the terms, we end up with the following final result:

$$
[B+\bar{B}+i(\bar{C} \dot{C}-\dot{\bar{C}} C)]\left[E \dot{E}+\frac{1}{2}\{B-\bar{B}-3 i(\dot{\bar{C}} C+\bar{C} \dot{C})\}\right]=0
$$

The above equation establishes the existence of the CFtype restriction $B+\bar{B}+i(\bar{C} \dot{C}-\dot{\bar{C}} C)=0$ on our theory due to the equivalence of the coupled (but equivalent) Lagrangians (i.e., $L_{B} \equiv L_{\bar{B}}$ ). This is due to the fact that, in no way, we can state the other combination $E \dot{E}+(1 / 2)\{B-\bar{B}-3 i(\dot{\bar{C}}$ $C+\bar{C} \dot{C})\}=0$. On the contrary, the CF-type restriction $B+$ $\bar{B}+i(\bar{C} \dot{C}-\dot{\bar{C}} C)=0$ has been proven from various angles (cf. Equation (36)).

We end this appendix with the concluding remark that we have derived the CF-type restriction on our theory from theoretical requirements related to the symmetries of the coupled (but equivalent) Lagrangians and the absolute anticommutativity properties. However, our present derivation of the CF-type restriction is more direct as well as transparent.

B. On the Derivation of $f_{3}=E \dot{C}+\dot{E} C$ and $f_{2}=\dot{\bar{B}}$ $C-\bar{B} \dot{C}$

The theoretical content of this appendix is, first of all, devoted to the explicit derivation of $f_{3}(\tau)$ in the expansion 
of $\Sigma(\tau, \bar{\theta})$ in the antichiral super expansions (41). Towards this objective in mind, we focus on Equation (51) where the first-order differential equation w.r.t. the evolution parameter $\tau$ for $f_{3}$ has been expressed. We can rewrite it as

$f_{3} \dot{C}+\dot{f}_{3} C-\dot{E} \dot{C} C-E \ddot{C} C=0 \Rightarrow \frac{d}{d \tau}\left[f_{3} C\right]-\frac{d}{d \tau}[(\dot{E} C+E \dot{C}) C]=0$,

where we have used the fermionic property $\left(C^{2}=0\right)$ of the ghost variable $(C)$. The above equation can be reexpressed in a different but useful form as the total derivative w.r.t. $\tau$ :

$$
\frac{d}{d \tau}\left[\left\{f_{3}-(E \dot{C}+\dot{E} C)\right\} C\right]=0
$$

Integrating the above equation from $\tau=-\infty$ to $\tau=+\infty$ (which are the limiting cases for $\tau$ in our theory), we obtain the following relationship:

$$
\left[f_{3}-(E \dot{C}+\dot{E} C)\right] C=0
$$

We would like to point out that, while deriving (B.3) from (B.2), we have assumed that all the physical variables of the Lagrangian $L_{B}$ and the secondary variable $f_{3}(\tau)$ vanish off at $\tau= \pm \infty$. For $C \neq 0$, we obtain the desired result $f_{3}=E \dot{C}$ $+\dot{E} C$. We have taken $C \neq 0$ because the whole set of BRST symmetry transformations in Equation (33) is true only when the ghost variable $C(\tau)$ has the nontrivial and nonzero value.

We now concentrate on the precise determination of $f_{2}$ $(\tau)$ of the super expansions (41). In other words, we wish to show that $f_{2}=\dot{\bar{B}} C-\bar{B} \dot{C}$. For this purpose, we note that we have a first-order differential equation w.r.t. the evolution parameter $\tau$ for the secondary variable $f_{2}(\tau)$ in Equation (49). This can be reexpressed as follows:

$$
f_{2} \dot{C}+\dot{f}_{2} C-2 f_{2} \dot{C}-2 \dot{\bar{B}} \dot{C} C+\dot{\bar{B}} \dot{C} C+\bar{B} \ddot{C} C=0
$$

where we have added and subtracted $f_{2} \dot{C}$ and $\dot{\bar{B}} \dot{C} C$. The above equation implies that we have now its modified form (with total derivatives) as

$$
\begin{aligned}
& \frac{d}{d \tau}\left[f_{2} C\right]-2\left(f_{2} \dot{C}+\dot{\bar{B}} \dot{C} C\right)+\frac{d}{d \tau}[\bar{B} \dot{C} C] \\
& \quad=0 \Rightarrow \frac{d}{d \tau}\left[f_{2} C+\bar{B} \dot{C} C\right]-2\left[\left(f_{2}-\dot{\bar{B}} C\right) \dot{C}\right] \\
& \quad=0 .
\end{aligned}
$$

Using the fermionic $\left(C^{2}=\dot{C}^{2}=0\right)$ property of the ghost variables $C$ and $\dot{C}$, we can recast the above equation in the following interesting form where $\left[f_{2}-(\dot{\bar{B}} C-\bar{B} \dot{C})\right]$ appears very nicely in the individual terms of the following difference, namely,

$$
\frac{d}{d \tau}\left[\left\{f_{2}-(\dot{\bar{B}} C-\bar{B} \dot{C})\right\} C\right]-2\left[\left\{f_{2}-(\dot{\bar{B}} C-\bar{B} \dot{C})\right\} \dot{C}\right]=0
$$

We can expand the total derivative in the first term to obtain

$$
\frac{d}{d \tau}\left[\left\{f_{2}-(\dot{\bar{B}} C-\bar{B} \dot{C})\right\}\right] C-\left[\left\{f_{2}-(\dot{\bar{B}} C-\bar{B} \dot{C})\right\} \dot{C}\right]=0
$$

Defining $f_{2}-(\dot{\bar{B}} C-\bar{B} \dot{C})=\chi$ leads us to the following:

$$
\left(\frac{d}{d \tau} \chi\right) C-\chi \dot{C}=0 \Rightarrow \dot{\chi} C=\chi \dot{C}
$$

Multiplying from the right by $C$ and taking into account the fermionic (i.e., $C^{2}=0$ ) nature of the ghost variable $C$, we obtain the following:

$$
0=\chi \dot{C} C \Rightarrow \chi=0 \quad(\text { for } C \dot{C} \neq 0)
$$

It should be noted that we have the off-shell nilpotent BRST symmetry transformation $s_{b} C=C \dot{C}$ (cf. Equation (33)). As a consequence, we have the combination of the variables $C \dot{C} \neq 0$. If the symmetry of a theory is the guiding principle behind its beauty, it is physically correct to assume that $s_{b} C=C \dot{C} \neq 0$. In fact, if we take $C \dot{C}=0$, the whole beauty and sacrosanct properties (i.e., off-shell nilpotency and absolute anticommutativity) of the (anti)BRST symmetry transformations of our present theory will be spoiled. As a consequence, the CF-type restriction will no longer remain (anti-)BRST invariant. It should be recalled, however, that we have invoked the CF-type restriction in proving the equivalence of the Lagrangians (cf. Equation (35)) w.r.t. the (anti-)BRST symmetry transformations. Hence, our conclusion in (B.9) is correct which leads to the derivation of $f_{2}=\dot{\bar{B}} C-\bar{B} \dot{C}$ from $\chi=0$.

\section{On the Proof of the Conservation Law}

We take up here the expression for the BRST charge $Q_{B}$ (cf. Equation (74)) that has been derived using the Noether theorem (cf. Section 7). We exploit the EL-EOM derived from the Lagrangian $L_{B}$ to recast the expression for $\dot{Q}_{B}$, namely,

$$
\begin{aligned}
\dot{Q}_{B}= & \frac{\dot{C} E}{2}\left(p_{x}^{2}+2 m p_{t}\right)+\frac{C \dot{E}}{2}\left(p_{x}^{2}+2 m p_{t}\right)+C E\left(p_{x} \dot{p}_{x}+m p_{t}\right) \\
& +2 E \dot{E} B \dot{C}+E^{2} B \dot{C}+E^{2} B \ddot{C}+\dot{E}^{2} B C+E \ddot{E} B C+E \dot{E} \dot{B} C \\
& +E \dot{E} B \dot{C}+2 B \dot{B} C-i \dot{B} \bar{C} \dot{C} C-i B \dot{\bar{C}} \dot{C} C-i B \bar{C} \ddot{C} C
\end{aligned}
$$


into the following form:

$$
\begin{aligned}
\dot{Q}_{B}= & -i E^{2} \ddot{\bar{C}} \dot{C} C-i E \dot{E} \dot{\bar{C}} \dot{C} C+3 E \dot{E} B \dot{C}+E^{2} B \ddot{C}+\dot{E}^{2} B C \\
& +E \ddot{E} B C+2 B \dot{B} C+B^{2} \dot{C}-i \dot{B} \bar{C} \dot{C} C-i B \dot{\bar{C}} \dot{C} C-i B \bar{C} \ddot{C} C
\end{aligned}
$$

where we have used the EL-EOMs from $L_{B}$ as

$$
\begin{gathered}
\dot{p}_{x}=0 \\
\dot{p}_{t}=0 \\
\frac{1}{2}\left(p_{x}^{2}+2 m p_{t}\right)=-\dot{B} E+i E \ddot{\bar{C}} C-i E \dot{\bar{C}} \dot{C}
\end{gathered}
$$

The expression (C.2) can be further changed to a reduced form as follows:

$$
\dot{Q}_{B}=i B \dot{\bar{C}} \dot{C} C-i \dot{B} \bar{C} \dot{C} C-i E \dot{E} \dot{\bar{C}} \dot{C} C-i E^{2} \ddot{\bar{C}} \dot{C} C
$$

if we use the EL-EOM from $L_{B}$ w.r.t. the variable $\bar{C}$ as

$$
2 \dot{B} C+B \dot{C}+3 E \dot{E} \dot{C}+E^{2} \ddot{C}+\dot{E}^{2} C+E \ddot{E} C-i \bar{C} \ddot{C} C-2 i \dot{\bar{C}} \dot{C} C=0
$$

The expression in (C.4) can be proven to be equal to zero by using the following EL-EOM from $L_{B}$ w.r.t. the variable $C$, namely,

$$
i \dot{B} \bar{C}-i B \dot{\bar{C}}+i E \dot{E} \dot{\bar{C}}+i E^{2} \ddot{\bar{C}}-\ddot{\bar{C}} \bar{C} C-2 \dot{\bar{C}} \bar{C} \dot{C}=0
$$

We end this appendix with the remark that, in an exactly similar fashion, we can prove the conservation law $\left(\dot{Q}_{\bar{B}}=0\right)$ of the anti-BRST charge (cf. Equation (74)) which has been derived by exploiting the theoretical tricks of the Noether theorem.

\section{Data Availability}

No data were used to support this study.

\section{Conflicts of Interest}

The authors declare that there are no conflicts of interest.

\section{Acknowledgments}

Two of us (AKR and AT) gratefully acknowledge the financial support from the BHU fellowship program of Banaras Hindu University (BHU), Varanasi (U.P.), under which the present investigation has been carried out.

\section{References}

[1] C. Becchi, A. Rouet, and R. Stora, "The Abelian Higgs Kibble model, unitarity of the S-operator," Physics Letters B, vol. 52, no. 3, pp. 344-346, 1974.
[2] C. Becchi, A. Rouet, and R. Stora, "Renormalization of the Abelian Higgs-Kibble model," Communications in Mathematical Physics, vol. 42, no. 2, pp. 127-162, 1975.

[3] C. Becchi, A. Rouet, and R. Stora, "Renormalization of gauge theories," Annals of Physics, vol. 98, no. 2, pp. 287-321, 1976.

[4] I. V. TyutinLebedev Institute PreprintReport Number: FIAN39 (1975) (unpublished), https://arxiv.org/abs/0812.0580.

[5] P. A. M. Dirac, Lectures on Quantum Mechanics, Belfer Graduate School of Science, Yeshiva University Press, New York, 1964.

[6] K. Sundermeyer, Constrained Dynamics: Lecture Notes in Physics, vol. 169, Springer-Verlag, Berlin, 1982.

[7] L. Bonora and R. P. Malik, "BRST, anti-BRST and gerbes," Physics Letters B, vol. 655, no. 1-2, pp. 75-79, 2007.

[8] L. Bonora and R. P. Malik, "BRST, anti-BRST and their geometry," Journal of Physics A: Mathematical and Theoretical, vol. 43, no. 37, p. 375403, 2010.

[9] G. Curci and R. Ferrari, "Slavnov transformations and supersummetry,” Physics Letters B, vol. 63, no. 1, pp. 91-94, 1976.

[10] J. Thierry-Mieg, "Geometrical reinterpretation of FaddeevPopov ghost particles and BRS transformations," Journal of Mathematical Physics, vol. 21, no. 12, article 2834, 2838 pages, 1980.

[11] M. Quirós, F. J. de Urries, J. Hoyos, M. L. Mazón, and E. Rodriguez, "Geometrical structure of Faddeev-Popov fields and invariance properties of gauge theories," Journal of Mathematical Physics, vol. 22, no. 8, pp. 1767-1774, 1981.

[12] R. Delbourgo and P. D. Jarvis, "Extended BRS invariance and OSp (4/2) supersymmetry," Journal of Physics A: Mathematical and General, vol. 15, pp. 611-625, 1982.

[13] L. Baulieu and J. Thierry-Mieg, "The principle of BRS symmetry: an alternative approach to Yang-Mills theories," Nuclear Physics B, vol. 197, no. 3, pp. 477-508, 1982.

[14] L. Bonora and M. Tonin, "Superfield formulation of extended BRS symmetry," Physics Letters B, vol. 98, no. 1-2, pp. 48-50, 1981.

[15] L. Bonora, P. Pasti, and M. Tonin, "Extended BRS symmetry in non-Abelian gauge theories," Il Nuovo Cimento A, vol. 64, no. 3, pp. 307-331, 1981.

[16] L. Bonora, P. Pasti, and M. Tonin, "Superspace approach to quantum gauge theories," Annals of Physics, vol. 144, no. 1, pp. 15-33, 1982.

[17] L. Alvarez-Gaume and L. Baulieu, "The two quantum symmetries associated with a classical symmetry," Nuclear Physics B, vol. 212, no. 2, pp. 255-267, 1983.

[18] R. P. Malik, "Abelian 2-form gauge theory: superfield formalism," The European Physical Journal C, vol. 60, no. 3, pp. 457-470, 2009.

[19] R. P. Malik, "Superfield approach to nilpotent symmetries for QED from a single restriction: an alternative to the horizontality condition," Journal of Physics A: Mathematical and Theoretical, vol. 39, no. 33, pp. 10575-10587, 2006.

[20] R. P. Malik, "An alternative to the horizontality condition in the superfield approach to BRST symmetries," European Physical Journal C: Particles and Fields, vol. 51, no. 1, pp. 169-177, 2007.

[21] R. P. Malik, "Superfield approach to symmetries for matter fields in Abelian gauge theories," Journal of Physics A: Mathematical and Theoretical, vol. 37, no. 19, pp. 5261-5274, 2004.

[22] L. Bonora, "BRST and supermanifolds," Nuclear Physics B, vol. 912, pp. 103-118, 2016. 
[23] B. Chauhan, A. Tripathi, A. K. Rao, and R. P. Malik, "Supervariable and BRST approaches to a toy model of reparameterization invariant theory," https://arxiv.org/abs/1912.12909.

[24] A. Tripathi, B. Chauhan, A. K. Rao, and R. P. Malik, "Reparameterization invariant model of a supersymmetric system: BRST and supervariable approaches," https://arxiv.org/abs/ 2010.02737.

[25] S. Kumar, B. Chauhan, and R. P. Malik, "(Anti-)chiral supervariable approach to nilpotent and absolutely anticommuting conserved charges of reparametrization invariant theories: a couple of relativistic toy models as examples," International Journal of Modern Physics A: Particles and Fields; Gravitation; Cosmology; Nuclear Physics, vol. 33, no. 22, article 1850133, 2018.

[26] A. Shukla, N. Srinivas, and R. P. Malik, "Nilpotent symmetries of a 4Dmodel of Hodge theory: augmented (anti-)chiral superfield formalism," Annals of Physics, vol. 394, pp. 98-119, 2018.

[27] B. Chauhan, S. Kumar, and R. P. Malik, "Nilpotent charges in an interacting gauge theory and an $\mathcal{N}=2$ SUSY quantum mechanical model: (anti-)chiral superfield approach," International Journal of Modern Physics A: Particles and Fields; Gravitation; Cosmology; Nuclear Physics, vol. 34, no. 24, article 1950131, 2019.

[28] B. Chauhan, S. Kumar, A. Tripathi, and R. P. Malik, "Modified 2D Proca theory: revisited under BRST and (anti-)chiral superfield formalisms," Advances in High Energy Physics, vol. 2020, Article ID 3495168, 38 pages, 2020.

[29] B. Chauhan, S. Kumar, and R. P. Malik, "(Anti-)chiral superfield approach to interacting Abelian 1-form gauge theories: nilpotent and absolutely anticommuting charges," International Journal of Modern Physics A: Particles and Fields; Gravitation; Cosmology; Nuclear Physics, vol. 33, no. 4, article 1850026, 2018.

[30] R. Brunetti and K. Fredenhagen, "Time of occurrence observable in quantum mechanics," Physical Review A, vol. 66, no. 4, article 044101, 2002.

[31] R. Banerjee, B. Chakraborty, and S. Gangopadhyay, "Noncommutativity and reparametrization symmetry," Journal of Physics A: Mathematical and General, vol. 38, no. 4, pp. 957-971, 2005.

[32] R. P. Malik, "Noncommutativity in a simple toy model," International Journal of Modern Physics A, vol. 22, no. 14n15, pp. 2643-2660, 2007.

[33] L. Bonora and R. P. Malik, "BRST and superfield formalisms: A review," 2021, BHU-CAS preprint (Communicated to the journal: Universe).

[34] R. P. Malik, "Nilpotent symmetries of a model of 2D diffeomorphism invariant theory: BRST approach," 2016, https:// arxiv.org/abs/1608.04627.

[35] A. Tripathi, A. K. Rao, and R. P. Malik, "Superfield approaches to a model of bosonic string: Curci-Ferrari type restrictions," 2021, https://arxiv.org/abs/2102.10606. 